\title{
Nonlinear frequency response analysis of forced periodic operation of non-isothermal CSTR with simultaneous modulation of inlet concentration and inlet temperature
}

\author{
Daliborka Nikolića $^{\mathrm{a}}$, Andreas Seidel-Morgenstern ${ }^{\mathrm{b}}$, Menka Petkovska ${ }^{\mathrm{c}, *}$ \\ ${ }^{a}$ University of Belgrade/Institute for Chemistry, Technology and Metallurgy, Njegoševa 12, 11000 Belgrade, Serbia \\ b Otto-von-Guericke University and Max-Planck Institute for Dynamics of Complex Technical Systems, Magdeburg, Germany \\ ${ }^{\mathrm{c}}$ University of Belgrade/Faculty of Technology and Metallurgy, Department of Chemical Engineering, Karnegijeva 4, 11120 Belgrade, Serbia
}

\section{H I G H L I G H T S}

- Evaluation of periodic operations of a non-isothermal CSTR based on NFR method.

- The analysis of a non-isothermal, homogeneous, simple nth order reaction in a CSTR.

- Simultaneous modulation of inlet concentration and inlet temperature is considered.

- The optimal phase difference for maximal increase of conversion is defined.

- The results are tested on three numerical examples (two oscillatory and one non-oscillatory).

\section{A R T I C L E I N F O}

\section{Article history:}

Received 30 December 2014

Received in revised form

21 April 2015

Accepted 2 June 2015

Available online 18 June 2015

\section{Keywords:}

Nonlinear dynamics

Mathematical modeling

Chemical reactors

Simulation

Non-isothermal CSTR

Two-input modulation

\begin{abstract}
A B S T R A C T
The nonlinear frequency response (NFR) method is applied for evaluation of possible improvement through simultaneous periodic modulation of two inputs of a non-isothermal continuously stirred tank reactor (CSTR) in which homogeneous $n$th order reaction $A \rightarrow \operatorname{product}(s)$ takes place. The two modulated inputs are the concentration of the reactant in the feed steam and the temperature of the feed stream. The cross asymmetrical second order FRF which correlates the outlet concentration with both modulated inputs is derived and analyzed. The optimal phase difference which should be used in order to maximize the conversion is determined. The method is tested on three numerical examples of non-isothermal CSTRs: (a) one which is oscillatory stable with strong resonant behavior, (b) one which is oscillatory stable with weak resonant behavior and (c) one which is nonoscillatory stable. Good agreement between the results of the approximate NFR method and the results of "exact" numerical integration is obtained except for the reactor with strong resonance for forcing frequencies which are close to the resonant frequency and for the reactor with weak resonant behavior for forcing frequency equal to the resonant one in case of high forcing amplitudes.
\end{abstract}

(c) 2015 Elsevier Ltd. All rights reserved.

\section{Introduction}

Forced periodic operations of non-isothermal CSTRs have been investigated in the past fifty years both for single and two-input modulation (Ritter and Douglas, 1970; Sinčić and Bailey, 1977; Sterman and Ydstie 1990a, 1990b, 1991; Lee and Bailey, 1980; Lee et al., 1980; Rigopoulos et al., 1988; Chen et al., 1994; Sidhu et al., 2007). The theoretical and experimental investigations have shown that, in some cases, significant enhancement in the reactor performance can be obtained by forced periodic operation.

\footnotetext{
* Corresponding author. Tel.: +381113303 610; fax: +381113370 387.

E-mail address: menka@tmf.bg.ac.rs (M. Petkovska).
} 
The process improvement caused by periodic modulation of one or more inputs is a consequence of the system nonlinearity. The improvements for highly nonlinear system or those which exhibit resonance might be significant (Ritter and Douglas, 1970).

It is possible that multi-input periodic operations improve the steady-state performance even when single-input perturbations have negligible or detrimental effect on the system performance (Sterman and Ydstie, 1990b).

The investigations of Parulekar (2003) demonstrated that the higher the number of inputs subject to periodic modulation, the better the process (reactor) performance can be. Additionally, in the same theoretical study it was concluded that an increase in the number of inputs perturbed led to a broadening of the regions in the operating parameter space where forced periodic operations are superior to operation at optimal steady state (Parulekar, 2003).

In our previous work, we have proposed the nonlinear frequency response (NFR) method for identification of candidate systems for process enhancement through periodic operation and an approximate estimation of the magnitude of such enhancement. The NFR method, which is applicable for weakly nonlinear systems, is based on Volterra series, generalized Fourier transform and the concept of higher order frequency response functions (FRFs) (Weiner and Spina, 1980).

Up to now, we have applied the nonlinear frequency response method to several generic examples of forced periodic operations of chemical reactors. In most cases the reactor was an isothermal or non-isothermal CSTR with a simple nth order irreversible chemical reaction:

\section{$A \rightarrow \operatorname{product}(s)$.}

Inlet concentration (Marković et al., 2008; Petkovska et al., 2010; Nikolić-Paunić and Petkovska, 2013; Nikolić et al., 2014a), flow-rate (Nikolić-Paunić and Petkovska, 2013; Nikolić et al., 2014a), inlet temperature and temperature of the cooling/heating medium (Nikolić et al., 2014b) were used as periodically modulated inputs, separately (Marković et al., 2008; Petkovska et al., 2010; Nikolić et al., 2014a, 2014b) or two of them simultaneously (Nikolić-Paunić and Petkovska, 2013).

An isothermal CSTR with a simple $n$th order heterogeneous reaction with inlet concentration modulation (Petkovska et al., 2010) was also investigated, as well as isothermal plug flow reactor (PFR) and isothermal dispersed flow tubular reactor (DFTR) with simple $n$th order reaction and inlet concentration modulation (Marković et al., 2008).

Recently, we applied the NFR method to analyzing periodically operated non-isothermal CSTRs, with single input modulations (Nikolić et al., 2014a, 2014b). In Part I (Nikolić et al., 2014a), the modulated input was the inlet concentration or flow-rate, and in Part II (Nikolić et al., 2014b) it was the temperature of the inlet reaction stream or the temperature of the heating/cooling fluid.

In this manuscript the NFR method is applied for evaluating periodic operations of a non-isothermal CSTR subject to modulation of two inputs. It is assumed that a simple $n$th order homogeneous reaction takes place. We analyze the case when the concentration of the inlet stream and its temperature are modulated simultaneously. In this way, the database of the derived FRFs related to the periodically operated non-isothermal CSTRs is enriched and this manuscript complements our previous papers (Nikolić et al., 2014a, 2014b) where single input modulations of the non-isothermal CSTRs were analyzed.

In the next section are given the basics regarding nonlinear frequency response, in general, and the NFR method for fast evaluation of periodic processes.

\section{Nonlinear frequency response method for evaluating periodic processes with two modulated inputs}

By definition, frequency response is the quasi-stationary response of a stable system to a periodic (sinusoidal or co-sinusoidal) input, imposed around a steady-state (Douglas, 1972). It has been widely used in many fields of engineering, in order to investigate and study system dynamics. For linear systems the relationship between the system output (the frequency response) and the input is well known, the output spectrum $Y(j \omega)$ being equal to the input spectrum $X(j \omega)$ multiplied by the system's frequency response function (FRF) $G(j \omega)$ (Lang et al., 2007). Unlike linear systems, the relationship between the input and output spectra of nonlinear systems is more complicated. One approach to study nonlinear systems in the frequency domain is based on the concept of higher order frequency response functions (FRFs). This approach extends the linear FRF concept to the nonlinear case for a wide class of nonlinear systems which can be described by the Volterra series model (Lang et al., 2007).

Frequency response of a weakly nonlinear system is a complex periodic function. It contains the basic harmonic, which has the same frequency as the input modulation, a DC (non-periodic) component, and an infinite number of higher harmonics (Douglas, 1972; Weiner and Spina, 1980). On the other hand, the nonlinear model $\boldsymbol{G}$ of a weakly nonlinear system in the frequency domain can be replaced by an infinite sequence of FRFs of different orders. These FRFs are directly related to the DC component and different harmonics of the response (Weiner and Spina, 1980).

In the case of a weakly nonlinear system with multiple inputs, several sets of FRFs need to be defined. Fig. 1 represents a block diagram of a weakly nonlinear system with two inputs and one output, for which it is necessary to define three sets of FRFs: two of them relating

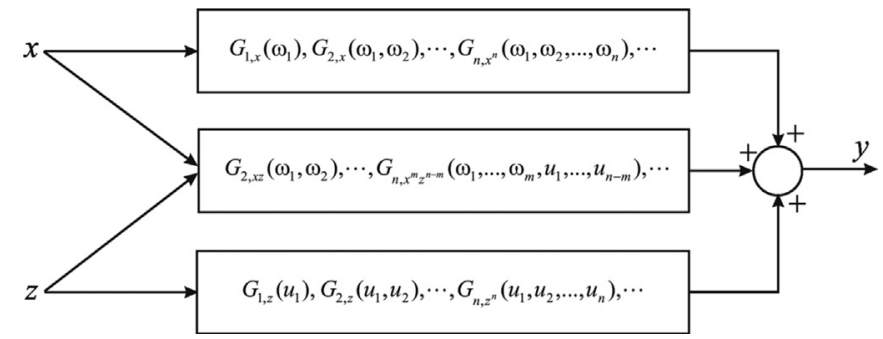

Fig. 1. Block diagram of a weakly nonlinear system with two inputs and one output. 
the output to each of the inputs, and one set of cross-functions, relating the output to both inputs. The third set contains only functions of the second and higher orders (Petkovska and Seidel-Morgenstern, 2013).

In Fig. 1 the following notation is used: $G_{n, x}^{n}$ and $G_{n, z}^{n}$ are the $n$ th-order FRFs corresponding to the individual inputs $x$ and $z$, while $G_{n, x}^{m-m}$ is the $n$ th-order cross-function, with order $m$ regarding the input $x$ and $n-m$, regarding the input $z$ (Petkovska and Seidel-Morgenstern, 2013).

For a weakly nonlinear system with two modulated inputs $x$ and $z$, the output of the system, $y$, is a sum of the contributions of the modulated inputs $x$ and $z$ separately (via the $G_{x}$ and $G_{z}$ functions), and the contribution corresponding to the cross-effect of both inputs (via the $G_{x z}$ functions). Each of these contributions can be presented as Volterra series (Petkovska and Seidel-Morgenstern, 2013).

$y(t)=y_{x}(t)+y_{z}(t)+y_{x z}(t)=\sum_{n=1}^{\infty} y_{x, n}(t)+\sum_{n=1}^{\infty} y_{z, n}(t)+\sum_{n=1}^{\infty} y_{x z, n}(t)$

The NFR method for fast evaluation of periodic operations, which is based on the nonlinear frequency response analysis of weakly nonlinear systems and the concept of higher order FRFs, has been explained in detail in our previous publications (Marković et al., 2008; Petkovska et al., 2010; Petkovska and Seidel-Morgenstern, 2013; Nikolić et al., 2014a, 2014b). The essence of the method is that the timeaverage performance of a periodic process is defined only by the DC component of the system's frequency response, which can be approximately estimated by using only the asymmetrical second order FRFs.

The details about application of the NFR method for systems with two modulated inputs can be found in Nikolić-Paunić and Petkovska (2013), where it was shown that the interaction of two modulated inputs will give the highest contribution to a possible process improvement when the two inputs are modulated with equal forcing frequencies. Here, we will repeat the main equations and conclusions about this case, as a non-isothermal CSTR with simultaneous modulation of the inlet concentration and temperature of the feed stream is in the focus of the current manuscript.

If two inputs $(x, z)$ are periodically modulated co-sinusoidal around a previously established steady-state $\left(x_{s}, z_{s}, y_{s}\right)$, with the same forcing frequency $\omega$, different forcing amplitudes $(A, B)$ and with a phase difference $(\varphi)$ between them:

$x(t)=x_{s}+A \cos (\omega t)$

$z(t)=z_{s}+B \cos (\omega t+\varphi)$

the DC components of the output $y$ consists of contributions of the modulations of inputs $x$ and $z$ separately and the cross-effect of both inputs

$y_{\mathrm{DC}}=y_{\mathrm{DC}, x}+y_{\mathrm{DC}, z}+y_{\mathrm{DC}, x z}$

By taking into account only the contribution of the second order FRFs, the DC components corresponding to the individual contributions of the inputs can be approximated by

$y_{\mathrm{DC}, x} \approx 2\left(\frac{A}{2}\right)^{2} G_{2, x x}(\omega,-\omega)$

$y_{\mathrm{DC}, z} \approx 2\left(\frac{B}{2}\right)^{2} G_{2, z z}(\omega,-\omega)$

The signs of the asymmetrical second-order FRFs $G_{2, x x}(\omega,-\omega)$ and $G_{2, z z}(\omega,-\omega)$ will define the signs of the DC components corresponding to the individual inputs (Marković et al., 2008).

The DC component which corresponds to the cross-effect of both inputs, taking into account only the second order FRF, is approximately

$y_{\mathrm{DC}, x z} \approx\left(\frac{A}{2}\right)\left(\frac{B}{2}\right)\left(e^{-j \varphi} G_{2, x z}(\omega,-\omega)+e^{j \varphi} G_{2, x z}(-\omega, \omega)\right)$

Considering that $G_{2, x z}(\omega,-\omega)$ and $G_{2, x z}(-\omega, \omega)$ are complex conjugates, by further transformation, the DC component which corresponds to the cross effect can also being written in the following form:

$y_{\mathrm{DC}, x z} \approx 2\left(\frac{A}{2}\right)\left(\frac{B}{2}\right)\left(\cos (\varphi) \operatorname{Re}\left(G_{2, x z}(\omega,-\omega)\right)+\sin (\varphi) \operatorname{Im}\left(G_{2, x z}(\omega,-\omega)\right)\right)$

After introducing the total asymmetrical second order cross term $G_{2, x z}^{*}$, which is a function of the forcing frequency and the phase difference between the modulated inputs:

$G_{2, x z}^{*}=\cos (\varphi) \operatorname{Re}\left(G_{2, x z}(\omega,-\omega)\right)+\sin (\varphi) \operatorname{Im}\left(G_{2, x z}(\omega,-\omega)\right)$

the DC component of the cross-effect can be written in the following form:

$y_{\mathrm{DC}, x z} \approx 2\left(\frac{A}{2}\right)\left(\frac{B}{2}\right) G_{2, x z}^{*}(\omega, \varphi)$

It is important to notice that the cross-effect of the modulation of two synchronized inputs strongly depends on the phase difference between them. As the matter of fact, the cross term can always be made negative, if that is desirable (e.g. if the output is defined as the outlet reactant concentration), by a proper choice of the phase difference $(\varphi)$. Furthermore, it is possible to determine the optimal phase difference for which the first derivative of the cross second order asymmetrical term $\partial G_{2, x z}^{*} / \partial \varphi$ is equal to zero, and consequently, the cross 
DC term has a minimum. This optimal phase difference is a function of the forcing frequency $\omega$ (Nikolić-Paunić and Petkovska, 2013):

$\varphi_{\text {opt }}=\operatorname{arctang}\left(\frac{\operatorname{Im}\left(G_{2, x z}(\omega,-\omega)\right)}{\operatorname{Re}\left(G_{2, x z}(\omega,-\omega)\right)}\right)-\pi$

Finally, when the two inputs are modulated with equal forcing frequencies $(\omega)$, different forcing amplitudes $(A, B)$ with a phase difference $(\varphi)$, the DC component of the output (Eq. (4)) can be approximately calculated using the single input and cross-asymmetrical second order FRFs, using the following expression:

$y_{\mathrm{DC}} \approx 2\left(\frac{A}{2}\right)^{2} G_{2, x x}(\omega,-\omega)+2\left(\frac{B}{2}\right)^{2} G_{2, z z}(\omega,-\omega)+2\left(\frac{A}{2}\right)\left(\frac{B}{2}\right) G_{2, x z}^{*}(\omega, \varphi)$

The procedure for deriving the higher order FRFs is standard and can be found in Petkovska (2001), Petkovska and Marković (2006), Marković et al. (2008), Petkovska et al. (2010), Petkovska and Seidel-Morgenstern (2013). The derivation process is recurrent, i.e., the firstorder FRFs have to be derived first, than the second order FRFs, etc. For this investigation, we limit our derivations and analysis to the firstorder and asymmetrical second-order FRFs.

In our previous publications we applied the NFR method for fast evaluation of chemical reactors (Marković et al., 2008; Petkovska et al., 2010; Petkovska and Seidel-Morgenstern, 2013; Nikolić-Paunić and Petkovska, 2013; Nikolić et al., 2014a, 2014b). It was explained that, for a reaction of the type $A \rightarrow \operatorname{product}(\mathrm{s})$, when one or more inputs are periodically modulated, the difference between the mean outlet concentration of the reactant $c_{A}^{m}$ and the corresponding steady-state outlet concentration $c_{A, s}\left(\Delta=c_{A}^{m}-c_{A, s}\right)$ defines whether the conversion can be increased by periodic operation. If $\Delta<0$, the periodic operation can be considered as favorable as it corresponds to increased conversion in comparison to the steady-state operation (Marković et al., 2008). It is important to point out that the difference $\Delta$ is equal to the DC component of the periodic change of the outlet reactant concentration, which can approximately be estimated from only the asymmetrical second order FRFs (Eq. (5) or (6) for single input modulation and Eq. (12) for simultaneous modulation of two inputs).

In principle, the NFR method should be used as a first step for fast screening of possible periodic operations, in order to detect processes which should further be investigated experimentally. It is meant to replace long and tedious numerical investigations. The most difficult and time consuming step of the NFR method is derivation of the needed FRFs, which needs to be performed only once. After that, all computations associated with the NFR method are reduced to simple algebra. So, the computational efforts of the NFR method are much less that those of the classical numerical investigations, which demand numerical integration of coupled sets of nonlinear differential equations. Furthermore, and what is more important, the NFR method gives a complete overview of the investigated periodic operation, with defined ranges of the forcing parameters (input frequency, amplitude(s) and phase differences, for cases of multiple modulated inputs) which should be used in order to obtain a favorable periodic operation. This is not possible with the classical numerical method, which gives results only for the defined sets of forcing parameters (frequency, amplitude and phase difference) for which numerical integrations are performed.

In this manuscript, we apply the NFR method for analysis of periodic operations of non-isothermal CSTRs with simple reaction mechanisms, for simultaneous modulation of the inlet concentration and inlet temperature. This application can be of great practical importance, as it can result with substantial improvement of the reactor performance, even in cases when separate modulation of inlet concentration or temperature give only small improvements or even reduce the reactor performance.

When applying the NFR method, the starting point is always the mathematical model of the investigated system. Therefore, the mathematical model of a non-isothermal CSTR will be presented in the next section.

\section{Mathematical model of a non-isothermal CSTR with simple reaction mechanism}

The mathematical model of a non-isothermal CSTR, in which a homogeneous $n$th order chemical reaction $A \rightarrow \operatorname{product}(s)$ takes place, is based on the assumptions that the reactor volume is constant and that the temperature of the heating/cooling fluid does not change from inlet to outlet. The well-known two nonlinear first order ODEs describing the material and energy balances can be found in Nikolić et al. (2014a). By changing the variables, the model can be transformed into dimensionless form, which can be even further transformed, by replacing all nonlinear terms with their Taylor series expansions (Nikolić et al., 2014a). Here we are giving just the final form, which is convenient for applying the NFR method (Nikolić et al., 2014a, 2014b):

$$
\frac{d C}{d \tau}=(1+\alpha)(\Phi+1)\left(C_{i}+1\right)-(\Phi+1)(C+1)-\alpha\left(1+n C+\gamma \theta+n \gamma C \theta+\left(\frac{\gamma^{2}}{2}-\gamma\right) \theta^{2}+\frac{1}{2} n(n-1) C^{2}+\cdots\right)
$$

Table 1

Definitions of the dimensionless variables.

\begin{tabular}{ll}
\hline Inlet concentration of the reactant & $C_{i}=\frac{C_{A, i}-C_{A i s}}{C_{A i s}}$ \\
Outlet concentration of the reactant & $C=\frac{c_{A}-C_{A, s}}{C_{A s}}$ \\
Inlet temperature & $\theta_{i}=\frac{T_{i}-T_{i s}}{T_{i s}}$ \\
Temperature in the reactor & $\theta=\frac{T-T_{s}}{T_{s}}$ \\
Temperature of the heating/cooling fluid & $\theta_{J}=\frac{T_{J}-T_{J . s}}{T_{J . s}}$ \\
Flow-rate & $\Phi=\frac{F-F_{s}}{F_{s}}$ \\
Time & $\tau=\frac{t}{V / F_{s}}$ \\
Frequency & $\omega=\omega_{d} \frac{V}{F_{s}}$ \\
\hline
\end{tabular}


$\frac{d \theta}{d \tau}=(1+\beta+S t-\delta)(\Phi+1)\left(\theta_{i}+1\right)-(\Phi+1)(\theta+1)-S t(\theta+1)+\delta\left(\theta_{J}+1\right)-\beta\left(1+n C+\gamma \theta+n \gamma C \theta+\left(\frac{\gamma^{2}}{2}-\gamma\right) \theta^{2}+\frac{1}{2} n(n-1) C^{2}+\cdots\right)$

In the dimensionless model Eqs. (13) and (14), $C$ and $C_{i}$ are the dimensionless concentrations of the reactant in the reactor and in the inlet stream, respectively, $\theta$ and $\theta_{i}$ are the dimensionless temperatures in the reactor and in the inlet stream, $\theta_{J}$ is the dimensionless temperature of the cooling/heating fluid, $\Phi$ is the dimensionless flow-rate and $\tau$ is the dimensionless time. Definitions of the dimensionless variables are given in Table 1, as well as the definition of a dimensionless frequency, which will be used in the frequency domain.

For the case of simultaneous periodic modulation of the inlet concentration and the inlet temperature the general dimensionless balance Eqs. (13) and (14) of the non-isothermal CSTR are reduced to the following model equations:

$\frac{d C}{d \tau}=(1+\alpha) C_{i}-(1+n \alpha) C-\alpha \gamma \theta-\alpha\left[\left(\frac{\gamma^{2}}{2}-\gamma\right) \theta^{2}+n \gamma C \theta+\frac{1}{2} n(n-1) C^{2}+\cdots\right]$

$\frac{d \theta}{d \tau}=(1+\beta+S t-\delta) \theta_{i}-(1+\beta \gamma+S t) \theta-n \beta C-\beta\left[\left(\frac{\gamma^{2}}{2}-\gamma\right) \theta^{2}+n \gamma C \theta+\frac{1}{2} n(n-1) C^{2}+\cdots\right]$

(the dimensionless flow-rate and the temperature of the cooling/heating fluid are equal to zero $\left(\Phi=0, \theta_{J}=0\right)$ ).

In Eqs. (13)-(16), $\alpha, \beta, \gamma, \delta$ and $S t$ are dimensionless auxiliary parameters, defined in the following way:

$\alpha=k_{0} e^{-\frac{E_{A}}{R T_{S}}} c_{A, S}^{n-1} \frac{V}{F_{S}}, \quad \beta=\frac{\Delta H_{R} k_{0} e^{-\frac{E_{A}}{R T_{S}}} c_{A, S}^{n}}{\rho c_{p} T_{S}} \frac{V}{F_{S}}, \quad \delta=\frac{U A_{w} T_{J, s}}{F_{S} \rho c_{p} T_{s}}, \quad \gamma=\frac{E_{A}}{R T_{s}}, \quad S t=\frac{U A_{w}}{F_{S} \rho c_{p}}$

Hereby the Stanton number $S t$ is the well know relative cooling intensity. In Table 1 and the definitions of the introduced auxiliary parameters (Eq. (17)), $c_{A}$ is the reactant concentration, $T$ temperature, $F$ flow-rate, $V$ the reactor volume, $k_{o}$ the preexponential factor in the Arrhenius equation, $E_{A}$ activation energy and $R$ the universal gas constant, $\Delta H_{r}$ heat of reaction, $U$ the overall heat transfer coefficient, $A_{W}$ the surface area for heat exchange, $\rho$ density, $c_{p}$ heat capacity and $\omega_{d}$ dimensional frequency. The subscripts are: $i$ for inlet, $s$ for steadystate and $J$ for the heating/cooling fluid (fluid in the jacket) (Nikolić et al., 2014a).

The auxiliary parameters (Eq. (17)) depend on the physical parameters of the system, and on the steady-state values of concentration, temperature, flow-rate and temperature of the heating/cooling fluid.

\section{Frequency response functions of a non-isothermal CSTR}

\subsection{Definitions of FRFs}

When inlet concentration and inlet temperature are periodically modulated, the non-isothermal CSTR represents a nonlinear system with two modulated inputs and two outputs, namely the outlet concentration and temperature. In order to describe the system, it is necessary to derive the following six sets of FRFs:

- Set 1: $G_{1, C}(\omega), G_{2, C C}(\omega,-\omega), \ldots-$ FRFs which correlate the dimensionless outlet concentration of the reactant with the modulated dimensionless inlet concentration.

- Set 2: $F_{1, C}(\omega), F_{2, c C}(\omega,-\omega), \ldots-$ FRFs which correlate the dimensionless outlet temperature with the modulated dimensionless inlet concentration.

- Set 3: $G_{1, T}(\omega), G_{2, T T}(\omega,-\omega), \ldots-F R F$ which correlate the dimensionless outlet concentration with the modulated dimensionless inlet temperature.

- Set 4: $F_{1, T}(\omega), F_{2, T T}(\omega,-\omega), \ldots-$ FRFs which correlate the dimensionless outlet temperature with the modulated dimensionless inlet temperature.

- Set 5: $G_{2, C T}(\omega,-\omega), G_{2, C T}(-\omega, \omega), \ldots-$ The cross FRFs which correlate the dimensionless outlet concentration with the modulated dimensionless inlet concentration and temperature.

- Set 6: $F_{2, C T}(\omega,-\omega), F_{2, C T}(-\omega, \omega), \ldots-$ The cross FRFs which correlate the dimensionless outlet temperature with the modulated dimensionless inlet concentration and temperature.

Since we are interested in the conversion change, the F-functions, which correlate the outlet temperature with the modulated input(s), are not subject of our interest. Still, the $F$-functions need to be derived since they are required in the derivation process of the $G$-functions. The derivation of the F-functions will be provided below, but without their further analysis.

\subsection{Derivation of the FRFS}

The basic steps of the procedure of derivation of the frequency response functions are:

1. The inlet concentration $C_{i}(\tau)$ and the inlet temperature $\theta_{i}(\tau)$ are defined in the form of co-sinusoidal functions with equal frequencies and a phase shift between them (analogous to Eqs. (2) and (3)),

2. The outlet concentration $C(\tau)$ and temperature $\theta(\tau)$ are expressed in the Volterra series form (analogous to Eq. (1)),

3. The expressions for $C_{i}(\tau), \theta_{i}(\tau), C(\tau)$ and $\theta(\tau)$ are substituted into the corresponding dimensionless model Eqs. (15) and (16), 
4. The method of harmonic probing is applied to the equations obtained in step 3 (the terms with the same amplitude and frequency are collected and equated to zero). For the derivation of the first order FRFs, the terms with $(A / 2) e^{j \omega \tau}$ and for the asymmetrical second order FRFs the terms with $(A / 2)^{2} e^{0}$ are collected and equated to zero.

5. The equations obtained in step 4 are solved.

The first order and asymmetrical second order FRFs for modulation of only the dimensionless inlet concentration have been derived in Nikolić et al. (2014a) and for modulation of the inlet temperature in Nikolić et al. (2014b). Here we are giving just the final expressions of the asymmetrical second order FRF which correlates the dimensionless outlet concentration with the modulated dimensionless inlet concentration:

$$
\begin{aligned}
G_{2, c c}(\omega,-\omega)= & -\frac{1}{2} \frac{\alpha(1+S t)}{1+n \alpha+\beta \gamma+n \alpha S t+S t} \\
& \times \frac{(1+\alpha)^{2}\left(n(n-1) \omega^{2}+\left[(1+S t)^{2}-2 \beta^{2} \gamma\right] n^{2}-\left[(1+S t+\beta \gamma)^{2}\right] n\right)}{\left[(1+n \alpha+\beta \gamma+n \alpha S t+S t)-\omega^{2}\right]^{2}+\omega^{2}(2+\beta \gamma+S t+n \alpha)^{2}}
\end{aligned}
$$

and the asymmetrical second order FRF which correlates the dimensionless outlet concentration with the modulated dimensionless inlet temperature:

$$
\begin{aligned}
G_{2, T T}(\omega,-\omega)= & -\frac{1}{2} \frac{\alpha \gamma(1+S t)(1+\beta+S t-\delta)^{2}}{(1+n \alpha+\beta \gamma+n \alpha S t+S t)} \\
& \times \frac{(\gamma-2) \omega^{2}-2 n^{2} \alpha^{2}-\alpha(4+\alpha \gamma) n+(\gamma-2)}{\left[(1+n \alpha+\beta \gamma+n \alpha S t+S t)-\omega^{2}\right]^{2}+\omega^{2}(2+\beta \gamma+S t+n \alpha)^{2}}
\end{aligned}
$$

The final expression for the cross-asymmetrical second order FRF $G_{2, C T}(\omega,-\omega)$ is given below:

$$
\begin{aligned}
G_{2, C T}(\omega,-\omega)= & \frac{n \alpha \gamma(1+\alpha)(1+S t)(1+\beta+S t-\delta)}{(n \alpha \beta \gamma-(1+n \alpha)(1+\beta \gamma+S t))\left(\left((1+n \alpha+\beta \gamma+n \alpha S t+S t)-\omega^{2}\right)^{2}+\omega^{2}(2+\beta \gamma+S t+n \alpha)^{2}\right)} \\
& \times\left(\left(1+S t+\alpha(1+S t+\beta \gamma)+2 \beta(1+n \alpha)+\omega^{2}\right)+j \omega(\alpha-2 \beta-S t)\right)
\end{aligned}
$$

Some details of the derivation procedure can be found in Appendix A.

\begin{tabular}{|c|c|c|}
\hline Condition & Frequency range & Sign of $G_{2, c c}(\omega,-\omega)$ \\
\hline$n=0$ & $\forall \omega$ & 0 \\
\hline$n<n_{C}$ and $n<0$ & $\forall \omega$ & Negative \\
\hline$n<n_{C}$ and $0<n<1$ & $\forall \omega$ & Positive \\
\hline$n_{C}<1$ and $n=1$ & $\forall \omega$ & Negative \\
\hline \multirow[t]{2}{*}{$n<n_{C}$ and $n>1$} & $\omega<\omega_{C}$ & Positive \\
\hline & $\omega>\omega_{C}$ & Negative \\
\hline \multirow[t]{2}{*}{$n>n_{C}$ and $n<0$} & $\omega<\omega_{C}$ & Positive \\
\hline & $\omega>\omega_{C}$ & Negative \\
\hline \multirow[t]{2}{*}{$n>n_{C}$ and $0<n<1$} & $\omega<\omega_{C}$ & Negative \\
\hline & $\omega>\omega_{C}$ & Positive \\
\hline$\frac{1}{n_{C}}<1$ and $n=1$ & $\forall \omega$ & Positive \\
\hline$n>n_{C}$ and $n>1$ & $\forall \omega$ & Negative \\
\hline
\end{tabular}

$G_{2, C T}(-\omega, \omega)$ is the conjugated complex function of the FRF $G_{2, C T}(\omega,-\omega)$

The real and imaginary parts of the cross-asymmetrical second-order FRF $G_{2, C T}(\omega,-\omega)$ are as follows:

$\operatorname{Re}\left(G_{2, C T}(\omega,-\omega)\right)=\frac{n \alpha \gamma(1+\alpha)(1+S t)(1+\beta+S t-\delta)}{n \alpha \beta \gamma-(1+n \alpha)(1+\beta \gamma+S t)}$

\begin{tabular}{|c|c|c|c|}
\hline Reaction order, $n$ & Sign of $(\gamma-2)$ & Forcing frequency, $\omega$ & Sign of $G_{2, T T}(\omega,-\omega)$ \\
\hline \multirow[t]{3}{*}{$n<n_{T 1}$ or $n>n_{T 2}$} & Positive & $\omega<\omega_{T}$ & Positive \\
\hline & & $\omega>\omega_{T}$ & Negative \\
\hline & Negative or zero & $\forall \omega$ & Positive \\
\hline \multirow[t]{3}{*}{$n_{T 1}<n<n_{T 2}$} & Negative & $\omega<\omega_{T}$ & Negative \\
\hline & & $\omega>\omega_{T}$ & Positive \\
\hline & Positive or zero & $\forall \omega$ & Negative \\
\hline \multirow[t]{3}{*}{$n=n_{T 1}$ or $n=n_{T 2}$} & Positive & $\forall \omega$ & Negative \\
\hline & Negative & $\forall \omega$ & Positive \\
\hline & Zero & $\forall \omega$ & Zero \\
\hline
\end{tabular}

Table 2

A summary of the results of the sign analysis for $G_{2, c c}(\omega,-\omega)$ (negative sign is desirable) (Reproduced from Nikolić et al., 2014a).

Table 3

Results of the sign analysis for $G_{2, T T}(\omega,-\omega)$ (negative sign is desirable) (Reproduced from Nikolić et al., 2014b). 


$$
\times \frac{\left(1+S t+\alpha(1+S t+\beta \gamma)+2 \beta(1+n \alpha)+\omega^{2}\right)}{\left((1+n \alpha+\beta \gamma+n \alpha S t+S t)-\omega^{2}\right)^{2}+\omega^{2}(2+\beta \gamma+S t+n \alpha)^{2}}
$$

$$
\begin{aligned}
\operatorname{Im}\left(G_{2, C T}(\omega,-\omega)\right)= & \frac{n \alpha \gamma(1+\alpha)(1+S t)(1+\beta+S t-\delta)}{n \alpha \beta \gamma-(1+n \alpha)(1+\beta \gamma+S t)} \\
& \times \frac{\omega(\alpha-2 \beta-S t)}{\left((1+n \alpha+\beta \gamma+n \alpha S t+S t)-\omega^{2}\right)^{2}+\omega^{2}(2+\beta \gamma+S t+n \alpha)^{2}}
\end{aligned}
$$

The final expressions for the cross-asymmetrical second order FRF, which correlates the dimensionless outlet concentration with both modulated inputs can be written as

$G_{2, C T}(\omega,-\omega)=\operatorname{Re}\left(G_{2, C T}(\omega,-\omega)\right)+j \operatorname{Im}\left(G_{2, C T}(\omega,-\omega)\right)$

The total asymmetrical second order cross term (Eq. (9)) is

$G_{2, C T}^{*}=\cos (\varphi) \operatorname{Re}\left(G_{2, C T}(\omega,-\omega)\right)+\sin (\varphi) \operatorname{Im}\left(G_{2, C T}(\omega,-\omega)\right)$

The optimal phase difference as a function of dimensionless forcing frequency, given by Eq. (11), after incorporating the real and imaginary parts of the cross-asymmetrical second order FRF becomes:

$\varphi_{\text {opt }}=\arctan \left(\frac{(\alpha-2 \beta-S t) \omega}{\omega^{2}+1+S t+\alpha(1+S t+\beta \gamma)+2 \beta(1+n \alpha)}\right)-\pi$

Table 4

Results of the sign analysis of the real part of $G_{2, C T}(\omega,-\omega)$.

\begin{tabular}{lll}
\hline Reaction order, $n$ & $\varepsilon_{R}$ & Forcing frequency, $\omega$ \\
\hline Zero & Any $\left(G_{2, C T}(\omega,-\omega)\right)$ & $\forall \omega$ \\
Positive & Positive or zero & $\forall \omega$ \\
& Negative & $\omega<\omega_{0, C T}$ \\
& & $\omega=\omega_{0, C T}$ \\
Negative & Positive or zero & $\omega>\omega_{0, C T}$ \\
& Negative & $\forall \omega$ \\
& & $\omega<\omega_{0, C T}$ \\
Positive & $\omega=\omega_{0, C T}$ \\
Negative & $\omega>\omega_{0, C T}$ \\
\hline
\end{tabular}

\begin{tabular}{|c|c|c|}
\hline Reaction order, $n$ & $\varepsilon_{I}$ & $\operatorname{Im}\left(G_{2, C T}(\omega,-\omega)\right)$ \\
\hline Zero & Any & Zero \\
\hline \multirow[t]{3}{*}{ Positive } & Zero & Zero \\
\hline & Positive & Negative \\
\hline & Negative & Positive \\
\hline \multirow[t]{3}{*}{ Negative } & Zero & Zero \\
\hline & Positive & Positive \\
\hline & Negative & Negative \\
\hline
\end{tabular}

Table 5

Results of the sign analysis of the imaginary part of $G_{2, C T}(\omega,-\omega)$.

\begin{tabular}{|c|c|c|}
\hline $\operatorname{Re}\left(G_{2, C T}(\omega,-\omega)\right)$ & $\operatorname{Im}\left(G_{2, C T}(\omega,-\omega)\right)$ & $\varphi$ \\
\hline Positive & Positive & $-\pi<\varphi<-\frac{\pi}{2}$ \\
\hline Positive & Negative & $\frac{\pi}{2}<\varphi<\pi$ \\
\hline Negative & Positive & $-\frac{\pi}{2}<\varphi<0$ \\
\hline Negative & Negative & $0<\varphi<\frac{\pi}{2}$ \\
\hline Positive & Zero & $\pi$ \\
\hline Zero & Positive & $-\frac{\pi}{2}$ \\
\hline Negative & Zero & 0 \\
\hline Zero & Negative & $\frac{\pi}{2}$ \\
\hline
\end{tabular}

Table 6

The range of the phase difference which assures negative sign of the cross term $G_{2, C T}^{*}$. 
Table 7

Model parameters for the numerical examples.

\begin{tabular}{|c|c|c|}
\hline \multicolumn{2}{|l|}{ Parameter } & Value \\
\hline \multicolumn{2}{|l|}{ Reaction order, $n$} & 1 \\
\hline \multicolumn{2}{|c|}{ Volume of the reactor, $V\left[\mathrm{~m}^{3}\right]$} & 1 \\
\hline \multicolumn{2}{|c|}{ Preexponential factor of the reaction rate constant, $k_{o},[1 / \mathrm{min}]$} & $1 * 10^{10}$ \\
\hline \multicolumn{2}{|c|}{ Activation energy, $E_{A}[\mathrm{~kJ} / \mathrm{kmol}]$} & 69,256 \\
\hline \multirow[t]{3}{*}{ Heat of reaction, $\Delta H_{r}[\mathrm{~kJ} / \mathrm{kmol}]$} & Numerical example 1 & $-543,920$ \\
\hline & Numerical example 2 & $-271,960$ \\
\hline & Numerical example 3 & $-54,392$ \\
\hline \multicolumn{2}{|l|}{ Heat capacity, $\overline{\rho c_{p}}\left[\mathrm{~kJ} / \mathrm{K} / \mathrm{m}^{3}\right]$} & $4.184 \times 10^{3}$ \\
\hline \multicolumn{2}{|c|}{ Steady-state flow-rate, $F_{s}\left[\mathrm{~m}^{3} / \mathrm{min}\right]$} & 1 \\
\hline \multicolumn{2}{|c|}{ Steady-state inlet concentration, $c_{A i, s}\left[\mathrm{kmol} / \mathrm{m}^{3}\right]$} & 2 \\
\hline \multicolumn{2}{|c|}{ Steady-state inlet temperature, $T_{i, s}[\mathrm{~K}]$} & 323 \\
\hline \multicolumn{2}{|c|}{ Steady-state temperature of the coolant, $T_{J, S}[\mathrm{~K}]$} & 365 \\
\hline \multicolumn{2}{|c|}{ Overall heat transfer coefficient multiplied by the heat transfer area, $U A_{W}[\mathrm{~kJ} / \mathrm{K} / \mathrm{min}]$} & 27,337 \\
\hline
\end{tabular}

Table 8

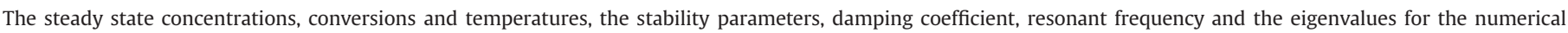
examples.

\begin{tabular}{|c|c|c|c|c|c|c|c|c|}
\hline \multirow[t]{2}{*}{ Numerical example } & \multicolumn{3}{|c|}{ Steady state point } & \multirow[t]{2}{*}{$A_{p s}$} & \multirow[t]{2}{*}{$B_{p s}$} & \multirow[t]{2}{*}{$\xi$} & \multirow[t]{2}{*}{$\omega_{r}$} & \multirow[t]{2}{*}{ The eigenvalues } \\
\hline & $c_{A, s}\left[\mathrm{kmol} / \mathrm{m}^{3}\right]$ & $x_{A, s}$ & $T_{s}[\mathrm{~K}]$ & & & & & \\
\hline 1 & 0.3466 & 0.8267 & 388.1 & -0.709 & 31.590 & 0.126 & 5.53 & $-0.709 \pm 5.576 i$ \\
\hline 2 & 0.7356 & 0.6322 & 370.5 & -2.632 & 15.495 & 0.669 & 1.28 & $-2.632 \pm 2.927 i$ \\
\hline 3 & 1.016 & 0.4920 & 361.3 & -4.343 & 14.016 & 1.160 & 1 & $-2.141,-6.544$ \\
\hline
\end{tabular}

\subsection{Stability and oscillatory analysis}

The NFR method is applicable only for stable systems. In Nikolić et al. (2014a), the stability conditions for the investigated system were derived and analyzed in detail. Let us just repeat here that the stability is determined by the characteristic equation of the system and its roots. For the non-isothermal CSTR defined by model Eqs. (15) and (16) the characteristic equation is the following second-order equation (Nikolić et al., 2014a):

$s^{2}+s(2+\beta \gamma+S t+n \alpha)+(1+n \alpha+\beta \gamma+n \alpha S t+S t)=0$

The analysis of the roots of this equation showed that the non-isothermal CSTR will be stable if the following conditions are met (Nikolić et al., 2014a):

$$
\begin{aligned}
& A_{p s}=-\frac{(2+n \alpha+S t+\beta \gamma)}{2}<0 \\
& B_{p s}=(1+n \alpha+\beta \gamma+n \alpha S t+S t)>0
\end{aligned}
$$

It was also shown that the system is oscillatory if $A_{p s}^{2}<B_{p s}$, otherwise the system is nonoscillatory. If the system is oscillatory, it can exhibit resonant behavior and the resonant frequency can also be calculated from the stability parameters $A_{p s}$ and $B_{p s}$, in the following way (Nikolić et al., 2014a):

$\omega_{r}=\sqrt{B_{p s}-2 A_{p s}^{2}}$

It can also be shown that the damping coefficient $\xi$ of the system can be calculated from the stability parameters using the following expression:

$\xi=-\frac{A_{p s}}{\sqrt{B_{p s}}}$

\section{Analysis of the signs of the asymmetrical second order functions}

\subsection{Asymmetrical second order FRFs $G_{2, C C}(\omega,-\omega)$ and $G_{2, T T}(\omega,-\omega)$}

The sign analysis of the asymmetrical second order FRFs, $G_{2, C C}(\omega,-\omega)$ and $G_{2, T T}(\omega,-\omega)$, were given in detail in Nikolić et al. (2014a, 2014b). Thus, here we repeat only the final results. 

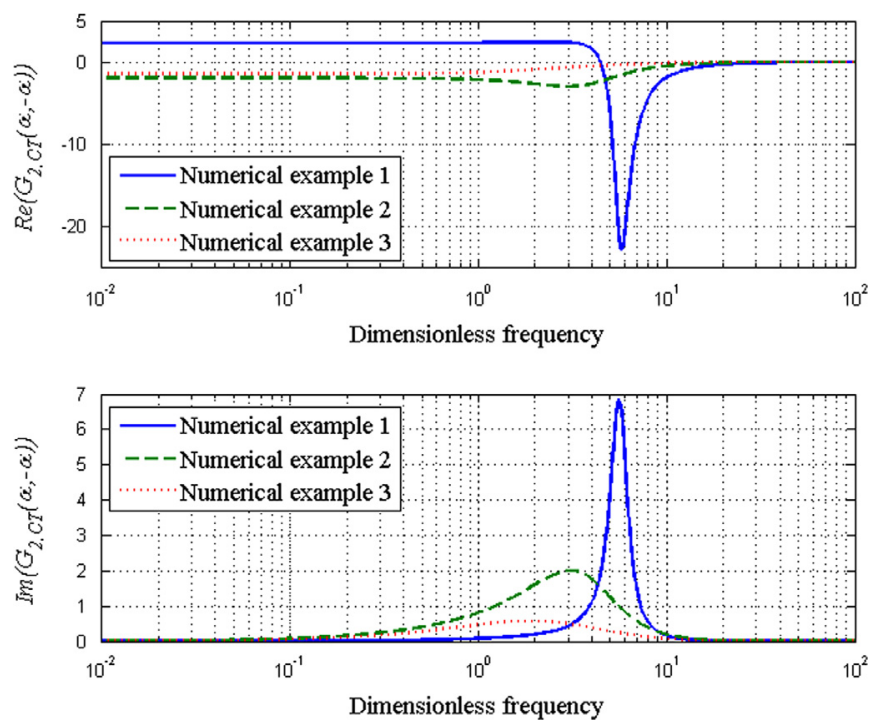

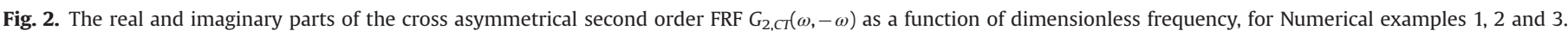

Table 9

The results of the sign analysis of the real and imaginary parts of $G_{2, C T}(\omega,-\omega)$, for the three numerical examples.

\begin{tabular}{|c|c|c|c|c|c|c|c|}
\hline Numerical example & $\varepsilon_{R}$ & $\omega_{0, C T}$ & Forcing frequency, $\omega$ & $\operatorname{Re}\left(G_{2, C T}(\omega,-\omega)\right)$ & $\varepsilon_{I}$ & $\operatorname{Im}\left(G_{2, C T}(\omega,-\omega)\right)$ & $\varphi$ \\
\hline 1 & -19.63 & 4.43 & $\begin{array}{l}\omega<4.43 \\
\omega=4.43 \\
\omega>4.43\end{array}$ & $\begin{array}{l}\text { Positive } \\
\text { Zero } \\
\text { Negative }\end{array}$ & -0.65 & Positive & $\begin{array}{l}-\pi<\varphi<-\frac{\pi}{2} \\
\varphi=-\frac{\pi}{2} \\
-\frac{\pi}{2}<\varphi<0\end{array}$ \\
\hline 2 & 10.70 & 1 & $\forall \omega$ & Negative & -4.37 & Positive & $-\frac{\pi}{2}<\varphi<0$ \\
\hline 3 & 13.90 & 1 & $\forall \omega$ & Negative & -5.50 & Positive & $-\frac{\pi}{2}<\varphi<0$ \\
\hline
\end{tabular}

In order to determine the sign of the asymmetrical second order FRF $G_{2, c c}(\omega,-\omega)$, it is necessary to calculate the following auxiliary parameter:

$n_{C}=\frac{(1+S t+\beta \gamma)^{2}}{(1+S t)^{2}-2 \beta^{2} \gamma}$

The asymmetrical second order FRF $G_{2, c c}(\omega,-\omega)$ can have the same sign in the whole frequency range or it can change its sign for a frequency given with the following equation:

$\omega_{C}=\sqrt{\frac{(1+S t+\beta \gamma)^{2}-n\left((1+S t)^{2}-2 \beta^{2} \gamma\right)}{n-1}}$

if $\omega_{C}$ is a real number.

The results of the sign analysis for $G_{2, C C}(\omega,-\omega)$, with respect to the reaction order $n$ and the calculated auxiliary parameters $\left(n_{C}, \omega_{C}\right)$ are summarized in Table 2 (Nikolić et al., 2014a).

In order to determine the sign of the asymmetrical second order FRF connected to the inlet temperature perturbation, $G_{2, T T}(\omega,-\omega)$, the following auxiliary parameters need to be calculated:

$n_{T 1, T 2}=\frac{-(4+\alpha \gamma) \pm \sqrt{\alpha^{2} \gamma^{2}+8 \alpha \gamma+\gamma}}{4 \alpha}$

The asymmetrical second order FRF $G_{2, T T}(\omega,-\omega)$ can also have the same sign in the whole frequency range or it can change its sign for a certain frequency, defined by the following equation:

$\omega_{T}=\sqrt{\frac{2 \alpha^{2} n^{2}+\alpha(4+\alpha \gamma) n-(\gamma-2)}{\gamma-2}}$

if $\omega_{T}$ is a real number.

The results of the sign analysis for the asymmetrical second order FRF $G_{2, T T}(\omega,-\omega)$ are given in Table 3, depending on the reaction order $n$ and the auxiliary parameters $n_{T 1}, n_{T 2}, \omega_{T}$ and $\gamma$ (Nikolić et al., 2014b). We assume that $n_{T 1}<n_{T 2}$. 


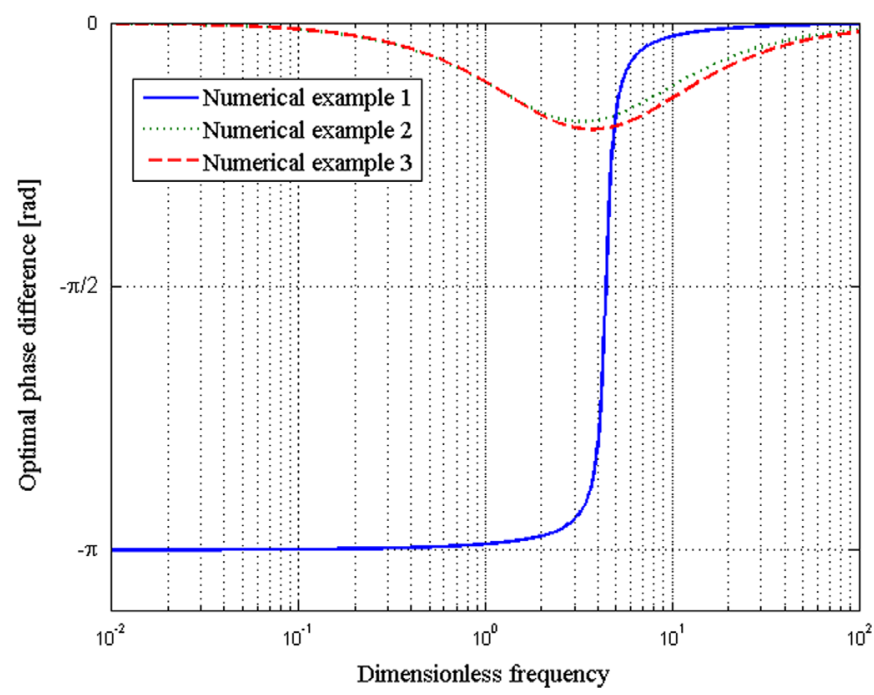

Fig. 3. The optimal phase differences as functions of dimensionless frequency, for Numerical examples 1, 2 and 3 .

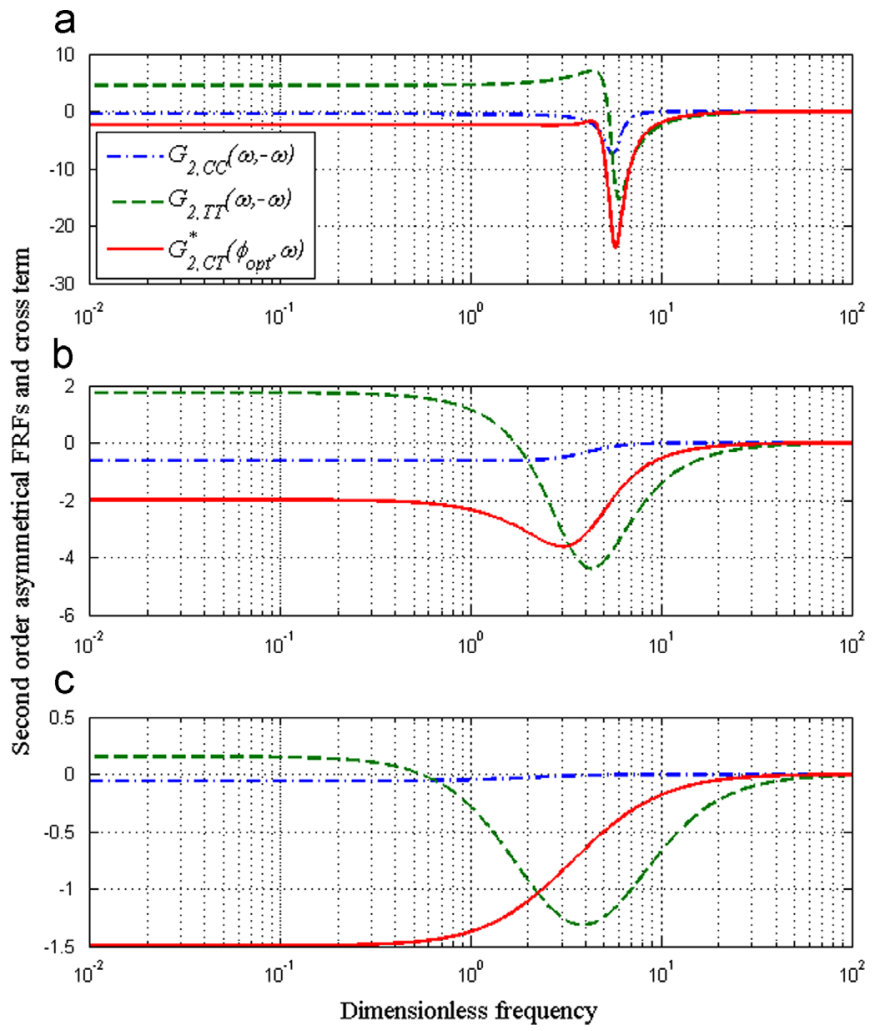

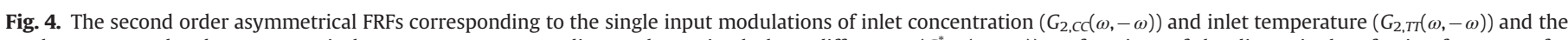

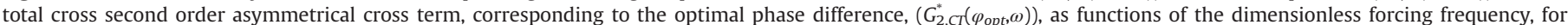
Numerical examples 1 (a), 2 (b) and 3 (c).

\subsection{The total asymmetrical second order cross term $G_{2, C T}^{*}$}

The sign of the total asymmetrical second order cross term $G_{2, C T}^{*}$ depends on the sign of the real and imaginary parts of the asymmetrical second order cross FRF $G_{2, C T}(\omega,-\omega)$ and the phase difference between the two modulated inputs (Eq. (24)).

After introducing the definitions of the stability parameter $A_{p s}$ and $B_{p s}$ in Eqs. (21) and (22), the real and imaginary parts of $G_{2, C T}(\omega,-\omega)$ can be rewritten in the following way:

$\operatorname{Re}\left(G_{2, C T}(\omega,-\omega)\right)=-\frac{n \alpha \gamma(1+\alpha)(1+S t)(1+\beta+S t-\delta)}{B_{p s}}$ 


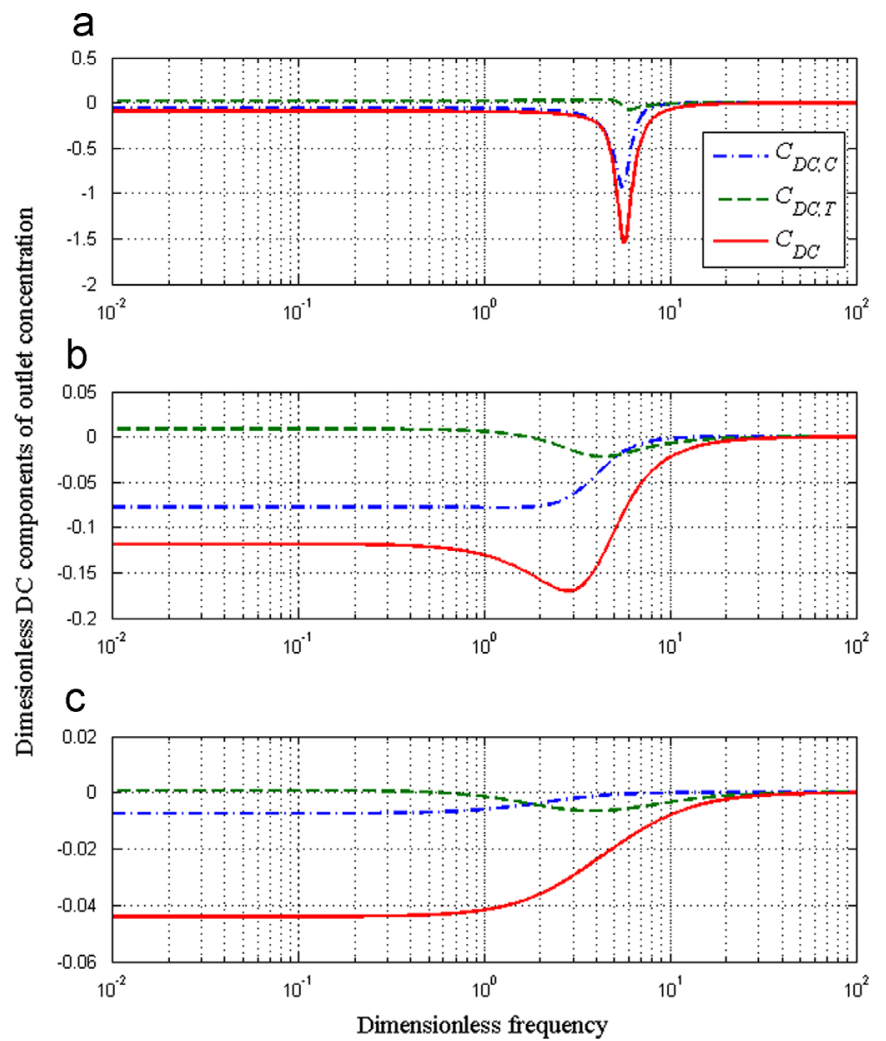

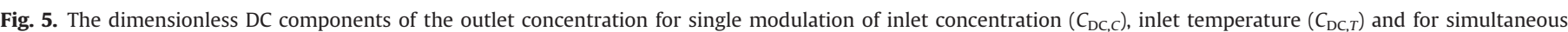

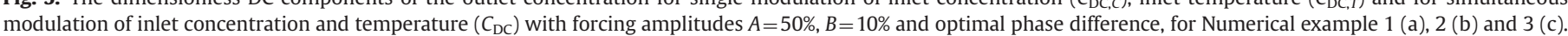

\section{Table 10}

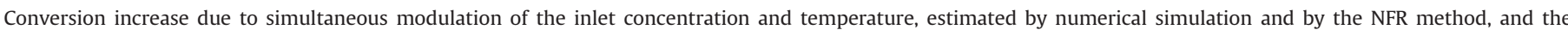
relative errors, for Numerical example 1.

\begin{tabular}{|c|c|c|c|c|c|c|c|c|c|c|}
\hline \multirow[t]{2}{*}{$\omega$} & \multirow[t]{2}{*}{$\varphi_{o p t}$} & \multicolumn{3}{|c|}{ Input amplitudes $A=50 \%, B=10 \%$} & \multicolumn{3}{|c|}{ Input amplitudes $A=25 \%, B=6 \%$} & \multicolumn{3}{|c|}{ Input amplitudes $A=10 \%, B=3 \%$} \\
\hline & & $\Delta x_{A, \text { num }}$ & $\Delta x_{A, N F R M}$ & $\delta_{c}$ & $\Delta x_{A, \text { num }}$ & $\Delta x_{A, N F R M}$ & $\delta_{c}$ & $\Delta x_{A, \text { num }}$ & $\Delta x_{A, N F R M}$ & $\delta_{c}$ \\
\hline 0.1 & -3.14 & 1.9930 & 1.9614 & -1.58 & 0.4988 & 0.4986 & -0.04 & 0.0789 & 0.0789 & 0.08 \\
\hline 1 & -3.11 & 2.0577 & 2.0400 & -0.86 & 0.5177 & 0.5176 & -0.01 & 0.0816 & 0.0817 & 0.11 \\
\hline 2 & -3.06 & 1.9917 & 2.3179 & 16.38 & 0.5786 & 0.5843 & 0.99 & 0.0913 & 0.0915 & 0.20 \\
\hline 3 & -2.96 & 1.3976 & 2.9706 & $>100$ & 0.5219 & 0.7393 & 41.66 & 0.1095 & 0.1139 & 3.96 \\
\hline 4 & -2.52 & 0.7775 & 4.7280 & $>100$ & 0.4697 & 1.1532 & $>100$ & 0.1498 & 0.1733 & 15.69 \\
\hline 5 & -0.55 & 2.1137 & 16.7432 & $>100$ & 0.6286 & 4.3432 & $>100$ & 0.2718 & 0.7300 & $>100$ \\
\hline 5.53 & -0.32 & 2.6064 & 31.8083 & $>100$ & 1.0774 & 8.6160 & $>100$ & 0.5145 & 1.5431 & $>100$ \\
\hline 6 & -0.24 & 2.8833 & 26.2170 & $>100$ & 1.4044 & 7.2957 & $>100$ & 0.6703 & 1.3550 & $>100$ \\
\hline 7 & -0.16 & 3.1206 & 8.5252 & $>100$ & 1.6224 & 2.4610 & 51.69 & 0.4450 & 0.4784 & 7.52 \\
\hline 8 & -0.12 & 2.8605 & 3.9032 & 36.45 & 1.0729 & 1.1512 & 7.30 & 0.2264 & 0.2295 & 1.39 \\
\hline 9 & -0.10 & 2.0949 & 2.2807 & 8.87 & 0.6665 & 0.6820 & 2.33 & 0.1371 & 0.1381 & 0.72 \\
\hline 10 & -0.08 & 1.4693 & 1.5232 & 3.67 & 0.4545 & 0.4598 & 1.19 & 0.0936 & 0.0941 & 0.50 \\
\hline
\end{tabular}

$$
\times \frac{\left(1+S t+\alpha(1+S t+\beta \gamma)+2 \beta(1+n \alpha)+\omega^{2}\right)}{\left[B_{p s}-\omega^{2}\right]^{2}+4 A_{p s}^{2} \omega^{2}}
$$

$\operatorname{Im}\left(G_{2, C T}(\omega,-\omega)\right)=-\frac{n \alpha \gamma(1+\alpha)(1+S t)(1+\beta+S t-\delta)}{B_{p s}}$

$$
\frac{\omega(\alpha-2 \beta-S t)}{\left[B_{p s}-\omega^{2}\right]^{2}+4 A_{p s}^{2} \omega^{2}}
$$

We can conclude from Eqs. (24), (34), (35) that the sign of the total cross asymmetrical second order term depends on:

- The parameters that are the characteristics of the system, i.e., the reaction order $n$ and auxiliary parameters $\alpha, \beta, \gamma, \delta$ (Eq. (17)) which are functions of the physical parameters of the reactor, the kinetics data of the chemical reaction and the steady-state concentration and temperature,

- The variables of the periodic operation, i.e., the forcing frequency $\omega$ and the phase difference between the two modulated inputs $\varphi$. 
Table 11

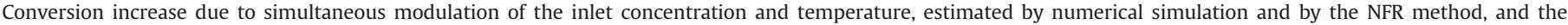
relative errors, for Numerical example 2.

\begin{tabular}{|c|c|c|c|c|c|c|c|c|c|c|}
\hline \multirow[t]{2}{*}{$\omega$} & \multirow[t]{2}{*}{$\varphi_{\text {opt }}$} & \multicolumn{3}{|c|}{ Input amplitudes $A=50 \%, B=10 \%$} & \multicolumn{3}{|c|}{ Input amplitudes $A=25 \% B=6 \%$} & \multicolumn{3}{|c|}{ Input amplitudes $A=10 \% B=3 \%$} \\
\hline & & $\Delta x_{A, \text { num }}$ & $\Delta x_{A, N F R M}$ & $\delta_{c}$ & $\Delta x_{A, \text { num }}$ & $\Delta x_{A, N F R M}$ & $\delta_{c}$ & $\Delta x_{A, \text { num }}$ & $\Delta x_{A, N F R M}$ & $\delta_{c}$ \\
\hline 0.1 & -0.04 & 6.4231 & 6.8851 & 7.19 & 1.7739 & 1.8103 & 2.05 & 0.3070 & 0.3080 & 0.30 \\
\hline 1 & -0.36 & 6.8433 & 7.6097 & 11.20 & 1.9671 & 2.0359 & 3.50 & 0.3534 & 0.3558 & 0.67 \\
\hline 1.28 & -0.43 & 7.0787 & 8.0260 & 13.38 & 2.0792 & 2.1672 & 4.23 & 0.3807 & 0.3840 & 0.87 \\
\hline 2 & -0.54 & 7.9070 & 9.2035 & 16.40 & 2.4382 & 2.5508 & 4.62 & 0.4644 & 0.4693 & 1.05 \\
\hline 3 & -0.59 & 9.1047 & 9.8570 & 8.26 & 2.7675 & 2.8257 & 2.11 & 0.5409 & 0.5443 & 0.62 \\
\hline 4 & -0.58 & 8.1281 & 8.3552 & 2.79 & 2.4392 & 2.4606 & 0.88 & 0.4887 & 0.4905 & 0.37 \\
\hline 5 & -0.55 & 5.9632 & 6.0454 & 1.38 & 1.8065 & 1.8169 & 0.58 & 0.3703 & 0.3713 & 0.26 \\
\hline 6 & -0.51 & 4.1630 & 4.2038 & 0.98 & 1.2770 & 1.2828 & 0.45 & 0.2662 & 0.2669 & 0.26 \\
\hline 7 & -0.47 & 2.9576 & 2.9799 & 0.75 & 0.9164 & 0.9197 & 0.36 & 0.1934 & 0.1939 & 0.24 \\
\hline 8 & -0.44 & 2.1750 & 2.1880 & 0.60 & 0.6792 & 0.6812 & 0.28 & 0.1447 & 0.1450 & 0.21 \\
\hline 9 & -0.41 & 1.6558 & 1.6638 & 0.48 & 0.5202 & 0.5214 & 0.24 & 0.1116 & 0.1118 & 0.20 \\
\hline 10 & -0.38 & 1.2993 & 1.3044 & 0.39 & 0.4100 & 0.4109 & 0.21 & 0.0885 & 0.0886 & 0.14 \\
\hline
\end{tabular}

Table 12

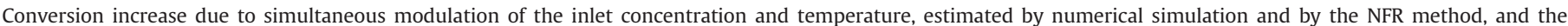
relative errors, for Numerical example 3.

\begin{tabular}{|c|c|c|c|c|c|c|c|c|c|c|}
\hline \multirow[t]{2}{*}{$\omega$} & \multirow[t]{2}{*}{$\varphi_{\text {opt }}$} & \multicolumn{3}{|c|}{ Input amplitudes $A=50 \%, B=10 \%$} & \multicolumn{3}{|c|}{ Input amplitudes $A=25 \%, B=6 \%$} & \multicolumn{3}{|c|}{ Input amplitudes $A=10 \%, B=3 \%$} \\
\hline & & $\Delta x_{A, \text { num }}$ & $\Delta x_{A, N F R M}$ & $\delta_{c}$ & $\Delta x_{A, \text { num }}$ & $\Delta x_{A, N F R M}$ & $\delta_{c}$ & $\Delta x_{A, \text { num }}$ & $\Delta x_{A, N F R M}$ & $\delta_{c}$ \\
\hline 0.1 & -0.04 & 4.5015 & 4.5462 & 0.99 & 1.3148 & 1.3210 & 0.47 & 0.2542 & 0.2551 & 0.37 \\
\hline 1 & -0.35 & 4.2607 & 4.3039 & 1.01 & 1.2629 & 1.2693 & 0.50 & 0.2494 & 0.2504 & 0.41 \\
\hline 2 & -0.55 & 3.6804 & 3.7055 & 0.68 & 1.1172 & 1.1217 & 0.40 & 0.2283 & 0.2292 & 0.42 \\
\hline 3 & -0.62 & 3.0319 & 3.0453 & 0.44 & 0.9386 & 0.9416 & 0.32 & 0.1970 & 0.1978 & 0.38 \\
\hline 4 & -0.63 & 2.4646 & 2.4731 & 0.34 & 0.7743 & 0.7763 & 0.26 & 0.1656 & 0.1662 & 0.34 \\
\hline 5 & -0.61 & 2.0064 & 2.0125 & 0.30 & 0.6371 & 0.6385 & 0.22 & 0.1381 & 0.1385 & 0.31 \\
\hline 6 & -0.58 & 1.6453 & 1.6495 & 0.26 & 0.5264 & 0.5275 & 0.21 & 0.1152 & 0.1155 & 0.31 \\
\hline 7 & -0.54 & 1.3616 & 1.3648 & 0.23 & 0.4380 & 0.4389 & 0.20 & 0.0965 & 0.0968 & 0.29 \\
\hline 8 & -0.51 & 1.1379 & 1.1405 & 0.23 & 0.3679 & 0.3684 & 0.11 & 0.0811 & 0.0816 & 0.67 \\
\hline 9 & -0.48 & 0.9610 & 0.9627 & 0.17 & 0.3114 & 0.3119 & 0.17 & 0.0691 & 0.0694 & 0.38 \\
\hline 10 & -0.45 & 0.8190 & 0.8204 & 0.17 & 0.2661 & 0.2665 & 0.16 & 0.0593 & 0.0595 & 0.35 \\
\hline
\end{tabular}

Since the forcing frequency and the phase difference are manipulated variables of the periodic operations, the sign analysis is performed in a way that, for a particular investigated system with defined parameters, the forcing frequency and the phase difference are determined for which the total cross asymmetrical second order function is negative $\left(G_{2, C T}^{*}<0\right)$.

\subsubsection{Sign of $\operatorname{Re}\left(G_{2, C T}(\omega,-\omega)\right)$}

The auxiliary parameters $\alpha, \gamma$ and $\delta$ are always positive, while $\beta$ can be positive (for endothermic reactions) or negative (for exothermic reactions). The stability conditions define the signs of stability parameters which have to be $A_{p s}<0$ and $B_{p s}>0$ for the system to be stable.

Considering this, we can conclude that the sign of the real part of the $G_{2, C T}(\omega,-\omega)$ function (Eq. (34)) depends on the reaction order $n$, the forcing frequency $\omega$ and the term

$\varepsilon_{R}=1+S t+\alpha(1+S t+\beta \gamma)+2 \beta(1+n \alpha)$

The real part of $G_{2, C T}(\omega,-\omega)$ changes its sign if $\varepsilon_{R}<0$, for a frequency

$\omega_{0, C T}=\sqrt{-\varepsilon_{R}}$

otherwise it has the same sign in the whole frequency range.

The results of the sign analysis of $\operatorname{Re}\left(G_{2, C T}(\omega,-\omega)\right)$ as a function of the reaction order $n$, auxiliary parameter $\varepsilon_{R}$, and forcing frequency are summarized in Table 4.

\subsubsection{Sign of $\operatorname{Im}\left(G_{2, C T}(\omega,-\omega)\right)$}

From the above mentioned observations and from Eq. (35), it can be concluded that the sign of $\operatorname{Im}\left(G_{2, C T}(\omega,-\omega)\right)$ depends on the reaction order $n$ and the term

$\varepsilon_{I}=\alpha-2 \beta-S t$

The final results of the sign analysis for the imaginary part of FRF $G_{2, C T}(\omega,-\omega)$, as a function of the reaction order $n$ and the sign of the term $\varepsilon_{I}$, are given in Table 5 . 


\subsubsection{Phase difference, $\varphi$}

For a particular system we can determine the signs of the real and imaginary parts of the asymmetrical cross second order FRF $G_{2}$, ${ }_{C T}(\omega,-\omega)$. If the real part changes sign for a certain frequency, the value of that forcing frequency can be calculated from Eq. (37).

The next step in the analysis is to choose the phase difference which should be used in order to ensure the negative sign of the total cross asymmetrical second order term $G_{2, C T}^{*}$.

The final results for the recommended phase difference which will assure that the cross term is negative are given in Table 6 .

It should be noted, that on the other hand, the optimal phase difference (Eqs. (11) and (25)), will always give the minimal possible value of the total cross second order asymmetrical term. Thus, the value of the optimal phase difference $\varphi_{\text {opt }}$ will always be in the recommended range of the phase difference.

\subsubsection{Sign of the DC component of the outlet concentration}

As concluded previously, it is always possible to achieve that the total asymmetrical second order cross term and the corresponding DC component originating from the cross-effect of both inputs $\left(C_{\mathrm{DC}, C T}\right)$ have negative signs, by appropriate choice of the phase difference.

However, in order to achieve increase of conversion through periodic operation, the total DC component of outlet concentration ( $\left.C_{\mathrm{DC}}\right)$ needs to be negative (Eq. (12)). Thus, it is necessary to consider the sign of the sum of the DC components corresponding to the individuals inputs $\left(C_{\mathrm{DC}, C}\right.$ and $\left.C_{, \mathrm{DC}, T}\right)$ and to the cross effect $\left(C_{\mathrm{DC}, C T}\right)$.

If the asymmetrical second order FRFs $G_{2, c}(\omega,-\omega)$ and $G_{2, T T}(\omega,-\omega)$ are both negative, it is obvious that simultaneous modulation of both inputs will ensure even higher conversion improvement. On the other hand if one of these FRFs is positive, than only evaluation of the total DC component can clarify the overall effect of the periodic operation on the reactor performance.

\section{Numerical examples}

\subsection{Definition of the numerical examples}

Simulation of the asymmetrical second order FRFs and the analysis whether, and to which extent, it would be possible to increase the conversion in a non-isothermal reactor owing to simultaneous modulation of the inlet concentration and inlet temperature is performed considering three numerical examples: one which corresponds to oscillatory stabile system with strong resonant behavior (Numerical example 1), one which corresponds to oscillatory stable system with weak resonant behavior (Numerical example 2) and one which corresponds to non-oscillatory stable system (Numerical example 3).

The model parameters corresponding to these numerical examples are given in Table 7. All parameters have the same values for all three numerical examples, except the heat of reaction. The steady state point defined with outlet concentration $\left(c_{A, s}\right)$, conversion $\left(x_{A, s}\right)$ and outlet temperature $\left(T_{s}\right)$ as well as the stability parameters $\left(A_{p s}, B_{p s}\right)$ (Eq. (27)), the damping coefficient $(\xi)$ (Eq. (29)), the resonant frequency $\left(\omega_{r}\right)$ (if existing) (Eq. (28)) and the eigenvalues, are given in Table 8, for all three numerical examples.

The conversion $x_{A, S}$ is defined in the standard way:

$x_{A, S}=\frac{c_{A i, s}-c_{A, s}}{c_{A i, S}}$

Numerical example 1 is identical to the one used for the cases of single inputs modulations (Nikolić et al., 2014a, 2014b). The reactor is oscillatory stable $\left(A_{p s}<0\right.$ and $\left.B_{p s}>0, A_{p s}^{2}<B_{p s}\right)$, with a low damping coefficient $\xi=0.126$ (Nikolić et al., 2014a). The non-isothermal CSTR defined as Numerical example 1 is highly nonlinear as a consequence of an assumed extremely high heat of reaction $\left(\Delta H_{r}=-543,920 \mathrm{~kJ} /\right.$ $\mathrm{kmol})$. Also, concerning that damping coefficient is quite low, the system is highly oscillatory with pronounced resonant behavior.

For Numerical example 2 the heat of reaction is 2 times lower than for the system defined as Numerical example 1 . The non-isothermal CSTR defined in this way is oscillatory stable with weak resonant behavior with the damping coefficient $\xi=0.669$.The increase of the damping coefficient for Numerical example 2 in comparison to the Numerical example 1 means that the system is less oscillatory.

Finally, Numerical example 3 corresponds to a non-isothermal CSTR with heat of the reaction which is 10 times lower than the heat of the reaction for Numerical example $1\left(\Delta H_{r}=-54,392 \mathrm{~kJ} / \mathrm{kmol}\right)$.The system is stable and non-oscillatory with damping coefficient $\xi=1.160$. The non-isothermal CSTR defined in this way does not exhibit resonant behavior.

The maximal allowed forcing amplitudes of the inlet concentration and inlet temperature are assumed to be the same for all numerical examples, $A_{\max }=100 \%, B_{\max }=15 \%$. The forcing amplitudes are not limited from the aspect of the system stability, but more from the aspect of what could be practically realized. E.g. the maximal amplitude of the inlet temperature corresponds to absolute maximal change in the inlet temperature of $\Delta T_{i, \max }=48.5 \mathrm{~K}$ (Nikolić et al., 2014a, 2014b).

\subsection{Simulation of the asymmetrical second order FRFs and the DC component of the outlet concentration}

In this section, the simulation results of the asymmetrical second order FRFs corresponding to the single input modulations, as well as the asymmetrical second order cross FRF and the DC component of the outlet concentration are presented for each numerical example.

A graphical representation of the real and imaginary parts of the cross second order asymmetrical FRF $G_{2, C T}(\omega,-\omega)$ as a function of dimensionless forcing frequency is given in Fig. 2 for all three numerical examples.

For Numerical example 1, the real and imaginary part of $G_{2, C T}(\omega,-\omega)$ both have extensive extremes near the resonant frequency $\left(\omega_{r}=5.53\right)$. For Numerical example 2, the real and imaginary parts of $G_{2, C T}(\omega,-\omega)$ again have extremes near the resonant frequency $\left(\omega_{r}=1.28\right)$, but not as big as for Numerical example 1. For the non-oscillatory stable non-isothermal CSTR, i.e., Numerical example 3, the real part of the cross asymmetrical second order FRF $G_{2, C T}(\omega,-\omega)$ has no extreme values and the imaginary part of this function has a maximum. 
The result of the sign analysis, in accordance with Tables 4 and 5 , as well as the values of the auxiliary parameters $\varepsilon_{R}$, $\omega_{0, C T}$ and $\varepsilon_{I}$ necessary for the sign analysis, are summarized in Table 9, with respect that the chemical reaction is first order ( $n=1)$. The results of the sign analysis of the real and imaginary parts of the asymmetrical second order cross FRF $G_{2, C T}(\omega,-\omega)$ are confirmed with simulation results, presented in Fig. 2.

The phase differences which should be used in order to achieve the negative value of the cross term $G_{2, \text {, } T}^{*}$ with respect to the signs of real and imaginary parts, according to Table 6, are also given in Table 9.

The optimal phase differences $\varphi_{\text {opt }}$ (defined by Eq. (25)), for all three numerical examples, are graphically presented in Fig. 3 . The optimal phase differences are in the ranges defined in Table 9.

The total asymmetrical second order cross term for the optimal phase difference $G_{2, C T}^{*}\left(\varphi_{\text {opt }}, \omega\right)$, as well as the asymmetrical second order FRFs which correspond to single input modulations $\left(G_{2, C C}(\omega,-\omega)\right.$ and $G_{2, T T}(\omega,-\omega)$, are graphically presented in Fig. 4(a)-(c), for Numerical examples 1, 2 and 3, respectively.

The asymmetrical second order FRFs which correspond to the single input modulations were analyzed and discussed in detail in Nikolić et al. (2014a, 2014b). Here, we will discuss only the phenomena related to simultaneous modulation of both inputs.

From Fig. 4 we can conclude the following:

- Similarly as for the single input modulations (Nikolić et al., 2014a, 2014b), the simultaneous modulation of inlet concentration and inlet temperature with high frequencies has no effect on the process performance, i.e.,

$\lim _{\omega \rightarrow \infty} G_{2, C T}^{*}=0$

- For low forcing frequencies, the total asymmetrical second order cross term has an asymptotic value which depends on the reaction order, dimensionless auxiliary parameters and the phase difference

$$
\lim _{\omega \rightarrow 0} G_{2, C T}^{*}=-\cos (\varphi) * \frac{n \alpha \gamma(1+\alpha)(1+S t)(1+\beta+S t-\delta)}{B_{p s}^{3}} \varepsilon_{R}
$$

For Numerical example 1, the low-frequency asymptotic value is -2.31 , for Numerical example $2,-1.99$ and for Numerical example 3 , -1.49 .

- For Numerical example 1, similarly to the asymmetrical second order FRFs which correspond to the single input modulations, the total asymmetrical second order cross term $G_{2, C T}^{*}\left(\varphi_{\text {opt }}, \omega\right)$ has an extensive minimum close to the resonant frequency $\omega_{r}=5.53$, where the highest improvement is expected. The minimal value of $G_{2, C T}^{*}\left(\varphi_{\text {opt }}, \omega\right)=-23.86$ is obtained for $\omega=5.74$ (Fig. $4(\mathrm{a})$ ).

- For Numerical example 2, the cross asymmetrical second order term $\left(G_{2, C T}^{*}\left(\varphi_{\text {opt }}, \omega\right)\right)$ has a minimum in the vicinity of the resonant frequency $\omega_{r}=1.28$, but not as extensive as for Numerical example 1 . The minimal value of $G_{2, C T}^{*}\left(\varphi_{\text {opt }}, \omega\right)=-3.62$ is obtained for $\omega=3.04$ (Fig. 4(b)).

- For the non-oscillatory non-isothermal CSTR defined as Numerical example 3, the total asymmetrical second order cross term $G_{2}^{*}$, $\left.{ }_{C T}\left(\varphi_{\text {opt }}, \omega\right)\right)$ has no extremes (Fig. 4(c)).

- For all three numerical examples, the asymmetrical second order FRF $G_{2, c c}(\omega,-\omega)$ is negative in the whole frequency range (which is in accordance with Table 2), while $G_{2, T T}(\omega,-\omega)$ changes its sign from positive to negative. $G_{2, T T}(\omega,-\omega)$ is positive for $\omega<\omega_{T}$ and negative for $\omega>\omega_{T}$ (Eq. (33), Table 3). The forcing frequency for which this FRF changes its sign is $\omega_{T}=5.24$ for Numerical example $1, \omega_{T}=1.82$ for Numerical example 2 and $\omega_{T}=0.87$ for Numerical example3.

- The asymmetrical second order cross term for the optimal phase $G_{2, C T}^{*}\left(\varphi_{\text {opt }}, \omega\right)$ is, as expected, negative in the whole frequency range for all three numerical examples (Fig. 4(a)-(c)).

- For forcing frequencies $\omega>\omega_{T}$ both asymmetrical second order FRFs corresponding to the single input modulations $G_{2, c c}(\omega,-\omega)$ and $G_{2}$, ${ }_{T T}(\omega,-\omega)$ are negative, which guaranties that in this frequency range simultaneous modulation of these two inputs will results with conversion enhancement, even higher than with single input modulations.

- For $\omega<\omega_{T}$, since the asymmetrical second order FRF $G_{2, T T}(\omega,-\omega)$ is positive, it is necessary to evaluate the total DC component of the outlet concentration in order to reveal whether improvement is possible in this frequency range.

The dimensionless DC components of the outlet concentration of the reactant A, as functions of dimensionless forcing frequency, are presented in Fig. 5, for all three numerical examples. Along with the total DC component obtained when the inlet concentration and inlet temperature are simultaneously modulated with the optimal phase difference $\left(C_{\mathrm{DC}}\right)$, the contributions to the DC component of the modulations of the inlet concentration $\left(C_{\mathrm{DC}, \mathrm{C}}\right)$ and inlet temperature $\left(C_{\mathrm{DC}, T}\right)$, separately, are also given. Fig. 5 was obtained for forcing amplitudes $A=50 \%, B=10 \%$.

From Fig. 5, it can be concluded that:

- For all three numerical examples, it is possible to achieve higher increase of conversion when both inputs are periodically modulated with optimal phase difference, in comparison to the single input modulations (with same forcing amplitudes).

- Even for forcing frequencies for which it is not possible to achieve higher conversion by modulation of the inlet temperature $\left(\omega<\omega_{T}\right.$, $G_{2, T T}(\omega,-\omega)>0$ ), if inlet concentration and inlet temperature are simultaneously modulated with forcing amplitudes $A=50 \%, B=10 \%$ and optimal phase difference, it is possible to achieve increase of conversion. This increase of conversion is higher in comparison to the single input modulation of inlet concentration.

- The dimensionless DC component of the outlet concentration for two-input modulation $\left(C_{\mathrm{DC}}\right)$ around the resonant frequency for Numerical example 2 is significantly lower that it is for Numerical example 1, around its resonant frequency. Nevertheless, the differences between the DC components for Numerical examples 1 and 2 at low-frequencies are much less significant than around the resonant frequencies. The dimensionless DC component of the outlet concentration for Numerical example 3 (non-oscillatory CSTR) is 
lower than the DC components for Numerical examples 1 and 2. This means that higher improvement can be expected for the highly nonlinear systems, which is in accordance with the previous investigations (Ritter and Douglas, 1970).

\subsection{Comparison with results obtained by numerical integration}

The conversion increase predicted by application of the NFR method is compared with the results obtained by numerical integration of the model equations for the periodic modulation of inlet concentration and inlet temperature with optimal phase difference. The model equations were numerically solved in their original, dimensional form (Nikolić et al., 2014a) by using a standard Matlab function ode15s.

The inputs were modulated in co-sinusoidal way around the previously established steady-state, in the following way:

$c_{A, i}(t)=c_{A i, s}\left(1+A * \cos \left(\omega_{d} t\right)\right)$

$T_{i}(t)=T_{i, s}\left(1+B * \cos \left(\omega_{d} t+\varphi_{\text {opt }}\right)\right)$

Based on the mean outlet concentration of the reactant $A, c_{A}^{m}$, calculated both from the numerical simulations and by applying the NFR method, the conversion of the reactant corresponding to the periodic process with simultaneous modulation of the inlet concentration and inlet temperature were calculated:

$x_{A, p}=\frac{c_{A i, s}-c_{A}^{m}}{c_{A i, s}}$

Than the increase of conversion owing to periodic operation, relative to the conversion in steady-state operation, was calculated:

$\Delta x_{A}(\%)=\frac{x_{A, p}-x_{A, S}}{x_{A, S}} * 100$

The results of numerical integration and of the NFR method are compared for 3 different combinations of forcing amplitudes, $A=50 \%$, $25 \%$ and $10 \%$ for inlet concentration (corresponding to absolute changes of inlet concentration of $1 \mathrm{kmol} / \mathrm{m}^{3}, 0.5 \mathrm{kmol} / \mathrm{m}^{3} \mathrm{and} 0.2 \mathrm{kmol} /$ $\mathrm{m}^{3}$, respectively) and $B=10 \%, 6 \%$ and $3 \%$ for inlet temperature (corresponding to absolute changes of inlet temperature of $32.3 \mathrm{~K}, 19.4 \mathrm{~K}$ and $9.7 \mathrm{~K}$, respectively) and for 12 different forcing frequencies, including the resonant frequency for the each numerical example (if existing).

In order to compare the agreement between the results obtained by the NFR method and by numerical integration, the relative errors were calculated, in the following way:

$\delta_{c}(\%)=\frac{\Delta x_{A, N F R M}-\Delta x_{A, \text { num }}}{\Delta x_{A, \text { num }}} * 100$

In Eq. (46) the value of the conversion change obtained by numerical simulation is considered to be exact.

The results of numerical integration and the corresponding results of the NFR method for Numerical example 1 are given in Table 10, for Numerical example 2 in Table 11 and for Numerical example 3 in Table 12. In Tables 10-12, the conversion increase and the relative error $\delta_{c}$ are given in percentages.

From the results given in Tables $10-12$, it can be concluded that:

- For Numerical example 1, good prediction by the NFR method is obtained only for frequencies which are not near to the resonant frequency. For lower forcing amplitudes the prediction is good in the wider range of forcing frequencies, closer to the resonant one.

- Numerical example 1 is identical to the numerical example used to test the quality of the NFR method for analysis of periodic operations of a non-isothermal CSTR with modulation of the inlet concentration (Nikolić et al., 2014a) and inlet temperature (Nikolić et al., 2014b), separately. It is important to notice that the quality of the NFR prediction of the process enhancement is about the same (the errors are in the same range), for the case of simultaneous modulation of both inputs, presented here, and for the cases of modulation of single inputs, presented in these references.

- For Numerical example 2, good prediction by the NFR method is obtained, except for the maximal amplitudes $(A=50 \%, B=10 \%)$ around the resonant frequency. For the forcing amplitudes $A=25 \%, B=6 \%$ and $A=10 \%, B=3 \%$, the relative errors are less than $5 \%$ even for forcing frequencies around the resonant one.

- For Numerical example 3, excellent agreement between the approximate (NFR method) and exact (numerical) solutions are obtained in the whole frequency range and for every combination of forcing amplitudes, even if they are very high. The maximal relative error is $1.01 \%$.

- The NFR method based on the second order approximation gives better prediction of the outlet concentration change for the oscillatory stable non-isothermal CSTR with weak resonant behavior (Numerical example 2) in comparison to the oscillatory stable nonisothermal CSTR with strong resonant behavior (Numerical example 1), which is highly nonlinear. The disagreements which were observed for the non-isothermal CSTRs which exhibit resonant behavior (Numerical examples 1 and 2) disappeared for the nonoscillatory non-isothermal CSTR (Numerical example 3).

\section{Conclusions}

In this paper the nonlinear frequency response method is used for evaluation of the possible improvement of non-isothermal CSTR with simple homogeneous $n$ th-order reaction, for the case of simultaneous periodic modulation of two inputs, namely concentration and temperature of the feed stream. The method is tested on three numerical examples of non-isothermal CSTRs: oscillatory stable reactor with strong resonant behavior and therefore highly non-linear (Numerical example 1), oscillatory stable reactor with weak resonant behavior (Numerical example 2) and non-oscillatory stable reactor (Numerical example 3). 
The main conclusions are that:

- The NFR method, based on the second order approximation, gives a correct answer whether the periodic operation of a non-isothermal CSTR with simultaneous modulation of the concentration and temperature of the feed stream is superior to the corresponding steadystate one (the sign of the predicted DC component is correct).

- The prediction of the process improvement through periodic operation is good, except around the resonant frequency, for the highly non-linear systems which exhibit strong resonant behavior (Numerical example 1). It should be noticed that the errors of the NFR prediction of the periodic operation with simultaneous modulation of the inlet concentration and temperature are similar as those obtained for single input modulations.

- By appropriate choice of the phase difference between the two modulated inputs, the cross effect of the two modulated inputs can be adjusted in the desired manner.

- Simultaneous modulation of the inlet concentration and temperature with optimal phase difference can give higher improvement than modulation of the two inputs, separately. Furthermore, even when modulation of the inlet temperature worsens the process (like for Numerical example 1, at low frequencies), simultaneous modulation of the inlet concentration and temperature results with process improvement.

In summary, we could say that the NFR method based on the second order approximation gives satisfactory results for over-damped and under-damped reactors with high and moderate damping coefficients, even for high input amplitudes, while it fails for low damping coefficients. The next step in our research will be to define exact criteria for the range of dumping coefficients for which the method gives reasonable approximations. This issue is directly related to defining the limitimg level of non-linearity and the acceptable range of input amplitudes for using the second order approximation and finding in which cases it would be necessary to introduce the forth, and possibly higher order FRFs, in order to withden that range. These issues need to be analysed together with analysis of convergence of the Volterra series expansion for the investigated system.

\section{Nomenclature}

A input amplitude

$A_{w} \quad$ surface area for heat exchange

$A_{p s} \quad$ stability parameter

$B \quad$ input amplitude

$B_{p s} \quad$ stability parameter

$c_{A} \quad$ reactant concentration

$c_{p} \quad$ heat capacity

C dimensionless concentration of reactant $\mathrm{A}$

$E_{A} \quad$ activation energy

F volumetric flow-rate

$F_{n} \quad n$ th-order frequency response function which correlates the dimensionless outlet temperature with the dimensionless modulated input(s), general

$G_{n} \quad n$ th-order frequency response function which correlates the dimensionless outlet concentration with the dimensionless modulated input(s), general

$G_{2, C T}^{*} \quad$ total asymmetrical second order cross term which correlates the dimensionless outlet concentration with both dimensionless modulated inputs (inlet concentration and inlet temperature)

$k_{o} \quad$ preexponential factor in Arrhenius equation

$n \quad$ reaction order

$R \quad$ universal gas constant

St Stanton number

$t$ time

$T \quad$ temperature

$U \quad$ overall heat transfer coefficient

$V \quad$ volume of the reactor

$x \quad$ input

$x_{A} \quad$ conversion of the reactant $\mathrm{A}$

$X \quad$ dimensionless input

$y \quad$ output

Y dimensionless output

$\mathrm{z} \quad$ input

Greek symbols

$\begin{array}{ll}\alpha & \text { auxiliary parameter } \\ \beta & \text { auxiliary parameter } \\ \gamma & \text { auxiliary parameter } \\ \delta & \text { auxiliary parameter } \\ \delta_{c} & \text { relative error }\end{array}$




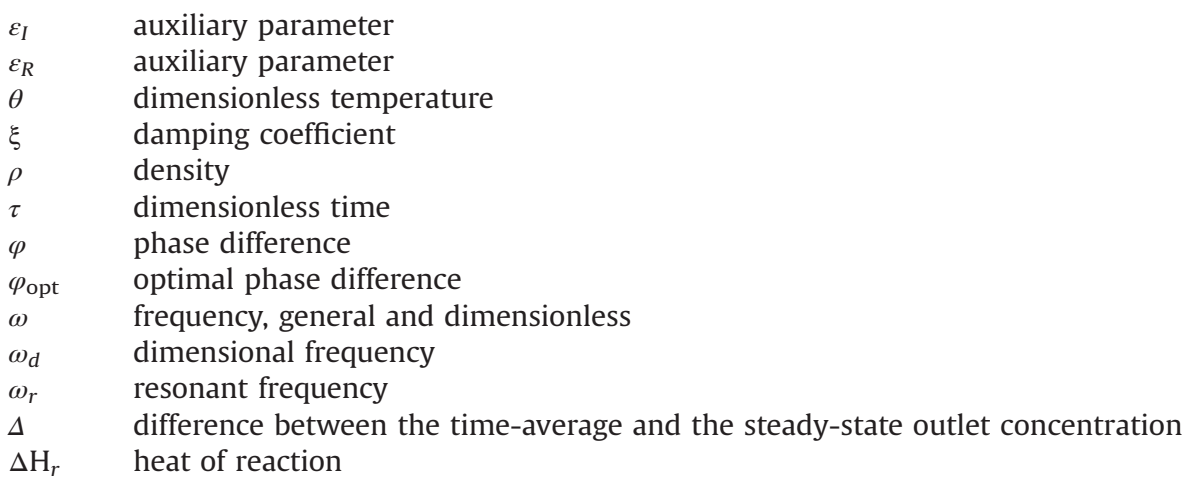

\section{Subscripts}

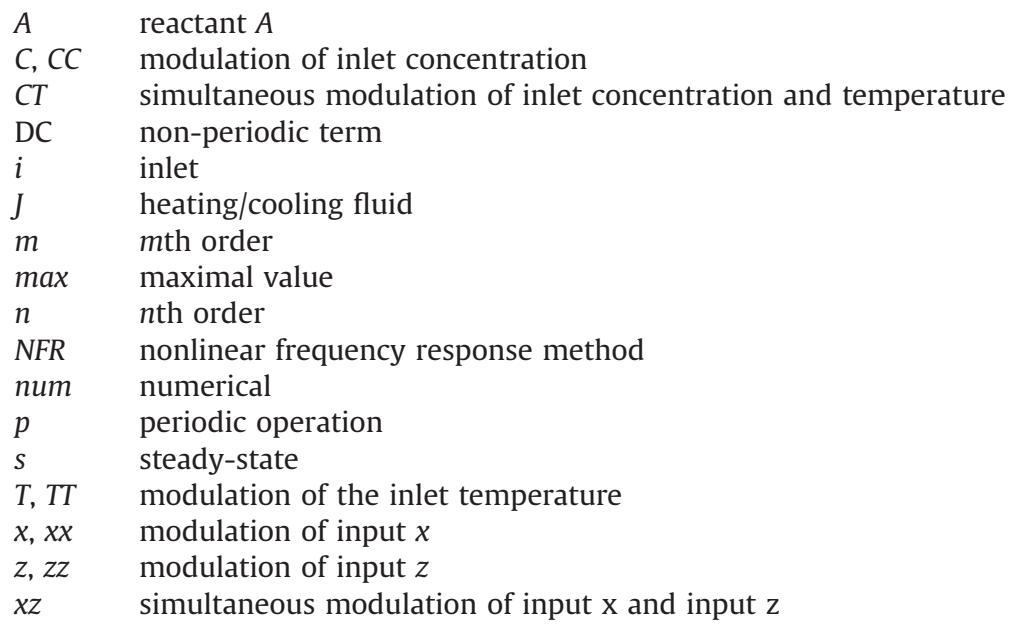

\section{Superscripts}

m mean

\section{Abbreviations}

CSTR continuous stirred tank reactor

FRF frequency response function

NFR nonlinear frequency response

\section{Acknowledgement}

This work was supported by the Serbian Ministry of Science in the frame of Projects nos. 172022 and III45001.

\section{Appendix A}

Derivation of the cross asymmetrical second order FRFs for simultaneous periodical modulation of inlet concentration and inlet temperature

Step 1: Defining the dimensionless inlet concentration and inlet temperature modulation, in the form of cosine functions:

$$
\begin{aligned}
& C_{i}(\tau)=A \cos (v \tau)=\frac{A}{2} e^{j v \tau}+\frac{A}{2} e^{-j v \tau} \\
& \theta_{i}(\tau)=B \cos (u \tau)=\frac{B}{2} e^{j u \tau}+\frac{B}{2} e^{-j u \tau}
\end{aligned}
$$


Step 2: Representing the dimensionless outlet concentration and dimensionless outlet temperature in the form of Volterra series:

$$
\begin{aligned}
C(\tau)= & \frac{A}{2} e^{j v \tau} G_{1, C}(v)+\frac{A}{2} e^{-j v \tau} G_{1, C}(-v)+\cdots+2\left(\frac{A}{2}\right)^{2} G_{2, C C}(v,-v) \\
& +\cdots+\frac{B}{2} e^{j u \tau} G_{1, T}(u)+\frac{B}{2} e^{-j u \tau} G_{1, T}(-u)+\cdots+2\left(\frac{B}{2}\right)^{2} G_{2, T T}(u,-u) \\
& +\cdots+\frac{A}{2} \frac{B}{2} e^{j(v+u) \tau} G_{2, C T}(v, u)+\cdots \\
\theta(\tau)= & \frac{A}{2} e^{j v \tau} F_{1, C}(v)+\frac{A}{2} e^{-j v \tau} F_{1, C}(-v)+\cdots \\
& +2\left(\frac{A}{2}\right)^{2} F_{2, C C}(v,-v)+\cdots+\frac{B}{2} e^{j u \tau} F_{1, T}(u)+\frac{B}{2} e^{-j u \tau} F_{1, T}(-u) \\
& +\cdots+2\left(\frac{B}{2}\right)^{2} F_{2, T T}(u,-u)+\cdots+\frac{A B}{2} e^{j(v+u) \tau} F_{2, C T}(v, u)+\cdots
\end{aligned}
$$

Step 3: Substituting the expressions for the dimensionless inlet concentration (Eq. (A1)), dimensionless inlet temperature (Eq. (A2)), dimensionless outlet concentration (Eq. (A3)) and dimensionless outlet temperature (Eq. (A4)), into the appropriate model equations (Eqs. (15) and (16)).

Step 4: After applying the method of harmonic probing, i.e., after collecting the terms with $(A / 2)(B / 2) e^{j(v+u) \theta}$, corresponding to the cross asymmetrical second order FRFs and equating them to zero, the following expressions are obtained:

$$
\begin{aligned}
j(v+u) G_{2, C T}(v, u)= & -(1+n \alpha) G_{2, C T}(v, u)-\alpha \gamma F_{2, C T}(v, u) \\
& -\alpha\left(\left(\gamma^{2}-2 \gamma\right) F_{1, C}(v) F_{1, T}(u)+n(n-1) G_{1, C}(v) G_{1, T}(u)+n \gamma G_{1, C}(v) F_{1, T}(u)+n \gamma G_{1, T}(u) F_{1, C}(v)\right) \\
j(v+u) F_{2, C T}(v, u)= & -(1+S t+\beta \gamma) F_{2, C T}(v, u) \\
& -n \beta G_{2, C T}(v, u)-\beta\left(\left(\gamma^{2}-2 \gamma\right) F_{1, C}(v) F_{1, T}(u)+n(n-1) G_{1, C}(v) G_{1, T}(u)+n \gamma G_{1, C}(v) F_{1, T}(u)+n \gamma G_{1, T}(u) F_{1, C}(v)\right)
\end{aligned}
$$

Step 5: Solving the equations obtained in step 4, Eq. (A5) and Eq. (A6), leads to the expression for the cross asymmetrical second order functions:

$G_{2, C T}(v, u)=\frac{\alpha \beta \gamma-\alpha(1+S t+\beta \gamma+j(v+u))}{(1+n \alpha+j(v+u))(1+S t+\beta \gamma+j(v+u))-n \alpha \beta \gamma}\left(\left(\gamma^{2}-2 \gamma\right) F_{1, C}(v) F_{1, T}(u)+n(n-1) G_{1, C}(v) G_{1, T}(u)+n \gamma G_{1, C}(v) F_{1, T}(u)+n \gamma G_{1, T}(u) F_{1, C}(v)\right)$

$F_{2, C T}(v, u)=\frac{n \alpha \beta-\beta(1+n \alpha+j(v+u)}{(1+n \alpha+j(v+u))(1+S t+\beta \gamma+j(v+u))-n \alpha \beta \gamma}\left(\left(\gamma^{2}-2 \gamma\right) F_{1, C}(v) F_{1, T}(u)+n(n-1) G_{1, C}(v) G_{1, T}(u)+n \gamma G_{1, C}(v) F_{1, T}(u)+n \gamma G_{1, T}(u) F_{1, C}(v)\right)$

After substituting the expressions for the first order FRFs (Nikolić et al., 2014a, 2014b) into Eq. (A7) and Eq. (A8) for the cross function for equal input frequencies, the following cross asymmetrical second order FRFs are obtained:

For $v=\omega$ and $u=-\omega$ :

$$
\begin{aligned}
G_{2, C T}(\omega,-\omega)= & \frac{n \alpha \gamma(1+\alpha)(1+S t)(1+\beta+S t-\delta)}{(n \alpha \beta \gamma-(1+n \alpha)(1+\beta \gamma+S t))\left(\left((1+n \alpha+\beta \gamma+n \alpha S t+S t)-\omega^{2}\right)^{2}+\omega^{2}(2+\beta \gamma+S t+n \alpha)^{2}\right)} \\
& \times\left(\left(1+S t+\alpha(1+S t+\beta \gamma)+2 \beta(1+n \alpha)+\omega^{2}\right)+j \omega(\alpha-2 \beta-S t)\right) \\
F_{2, C T}(\omega,-\omega)= & \frac{n \beta \gamma(1+\alpha)(1+\beta+S t-\delta)}{(n \alpha \beta \gamma-(1+n \alpha)(1+\beta \gamma+S t))\left(\left((1+n \alpha+\beta \gamma+n \alpha S t+S t)-\omega^{2}\right)^{2}+\omega^{2}(2+\beta \gamma+S t+n \alpha)^{2}\right)} \\
& \times\left(\left(1+S t+\alpha(1+S t+\beta \gamma)+2 \beta(1+n \alpha)+\omega^{2}\right)+j \omega(\alpha-2 \beta-S t)\right)
\end{aligned}
$$

For $v=-\omega$ and $u=\omega$ :

$$
\begin{aligned}
G_{2, C T}(-\omega, \omega)= & \frac{n \alpha \gamma(1+\alpha)(1+S t)(1+\beta+S t-\delta)}{(n \alpha \beta \gamma-(1+n \alpha)(1+\beta \gamma+S t))\left(\left((1+n \alpha+\beta \gamma+n \alpha S t+S t)-\omega^{2}\right)^{2}+\omega^{2}(2+\beta \gamma+S t+n \alpha)^{2}\right)} \\
& \times\left(\left(1+S t+\alpha(1+S t+\beta \gamma)+2 \beta(1+n \alpha)+\omega^{2}\right)-j \omega(\alpha-2 \beta-S t)\right) \\
F_{2, C T}(-\omega, \omega)= & \frac{n \beta \gamma(1+\alpha)(1+\beta+S t-\delta)}{(n \alpha \beta \gamma-(1+n \alpha)(1+\beta \gamma+S t))\left(\left((1+n \alpha+\beta \gamma+n \alpha S t+S t)-\omega^{2}\right)^{2}+\omega^{2}(2+\beta \gamma+S t+n \alpha)^{2}\right)} \\
& \times\left(\left(1+S t+\alpha(1+S t+\beta \gamma)+2 \beta(1+n \alpha)+\omega^{2}\right)-j \omega(\alpha-2 \beta-S t)\right)
\end{aligned}
$$

The cross asymmetrical second order FRFs $G_{2, C T}(\omega,-\omega)$ and $G_{2, C T}(-\omega, \omega)$ are complex conjugates, as well as $F_{2, C T}(\omega,-\omega)$ and $F_{2, C T}(-\omega, \omega)$. 


\section{References}

Chen, C.C., Hwang, C., Yang, R.Y.K., 1994. Optimal periodic forcing of nonlinear chemical processes for performance improvements. Can. J. Chem. Eng. 72, 672-682. Douglas, J.M., 1972. Process Dynamics and ControlPrentice-Hall, Englewood Cliffs, NJ.

Lang, Z.Q., Billings, S.A., Yue, J. Li, 2007. Output frequency response function of nonlinear Volterra systems. Automatica 43, 805-816.

Lee, C.K., Bailey, J.E., 1980. Modification of consecutive-competitive reaction selectivity by periodic operation. Ind. Eng. Chem. Process Des. Dev. $19,160-166$.

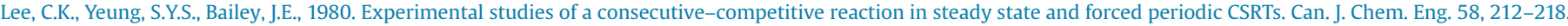

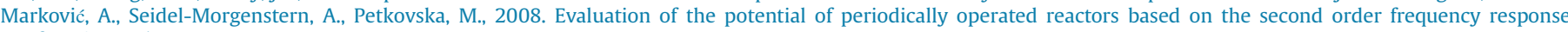
functions. Chem. Eng. Res. Des. 86, 682-691.

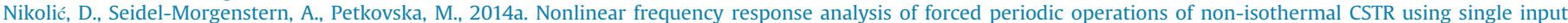
modulations. Part I: Modulation of inlet concentration or flow-rate. Chem. Eng. Sci. 117, 71-84.

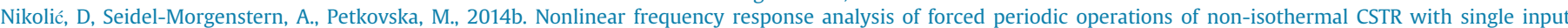
modulations. Part II: Modulation of inlet temperature or temperature of the cooling/heating fluid. Chem. Eng. Sci. 117, 31-44.

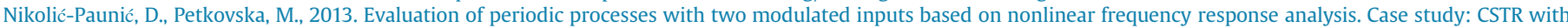
modulation of the inlet concentration and flow-rate. Chem. Eng. Sci. 104, 208-219.

Parulekar, S.J., 2003. Systematic performance analysis of continuous processes subject to multiple input cycling. Chem. Eng. Sci. 58, 5173-5194.

Petkovska, M., 2001. Nonlinear frequency response of non-isothermal adsorption systems. Nonlinear Dyn. $26,351$.

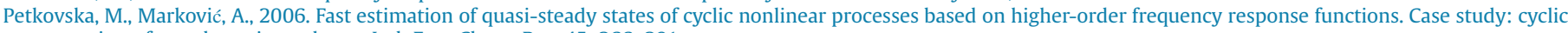
operation of an adsorption column. Ind. Eng. Chem. Res. 45, 266-291.

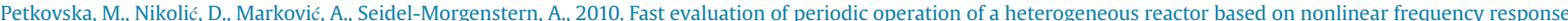
analysis. Chem. Eng. Sci. 65, 3632-3637.

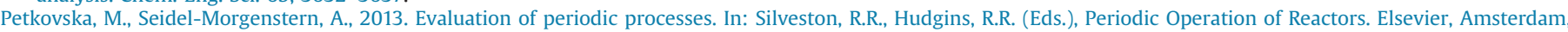
pp. 387-413.

Rigopoulos, K., Shu, X., Cinar, A., 1988. Forced periodic control of an exothermic CSTR with multiple input oscillations. AIChE J. 34, $2041-2051$.

Ritter, A.B., Douglas, J.M., 1970. Frequency response of nonlinear systems. Ind. Eng. Chem. Fundam. 9, 21-27.

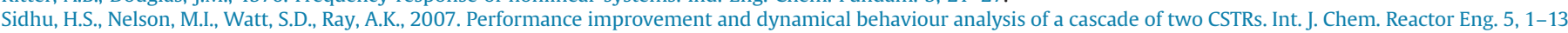
A13.

Sinčić, D., Bailey, J.E., 1977. Pathological dynamic behavior of forced periodic chemical processes. Chem. Eng. Sci. $32,281-286$.

Sterman, L.E., Ydstie, B.E., 1990a. The steady-state process with periodic perturbations. Chem. Eng. Sci. 45, 721-736.

Sterman, L.E., Ydstie, B.E., 1990b. Unsteady-state multivariable analysis of periodically perturbed systems. Chem. Eng. Sci. 45, 737-749.

Sterman, L.E., Ydstie, B.E., 1991. Periodic forcing of the CSTR: an application of the generalized П-criterion. AIChE J. 37, 986-996.

Weiner, D.D., Spina, J.F., 1980. Sinusoidal Analysis and Modeling of Weakly Nonlinear CircuitsVan Nostrand Reinhold Company, New York. 